\title{
23. LEG 55, EMPEROR SEAMOUNTS: TRACE ELEMENTS IN TRANSITIONAL THOLEIITES, ALKALI BASALTS, AND HAWAIITES-MANTLE HOMOGENEITY OR HETEROGENEITY AND MAGMATIC PROCESSES
}

\author{
P. Cambon, Centre Océanologique de Bretagne, CNEXO, B. P. 337, 29273 Brest cedex, France \\ J. L. Joron, Institut de Physique du Globe, 4, place Jussieu, 75230 Paris cedex 05, and Laboratoire P. Sue, \\ Centre d'Etudes Nucléaires, B. P. $n^{\circ} 2,91190$ Gif-sur-Yvette, France \\ H. Bougault, Centre Océanologique de Bretagne, CNEXO, B. P. 337, 29273 Brest cedex, France \\ M. Treuil, Institut de Physique du Globe, 4, place Jussieu, 75230 Paris cedex 05, and Laboratoire P. Sue, \\ Centre d'Etudes Nucléaires, B. P. nº 2, 91190 Gif-sur-Yvette, France
}

\section{INTRODUCTION}

Three seamounts of the Emperor Seamount chain were sampled during Leg 55:

Ōjin seamount, Hole 430A, $37^{\circ} 59.29 \mathrm{~N} 170^{\circ} 35.86 \mathrm{E}$ Nintoku seamount, Hole $432 \mathrm{~A}, 41^{\circ} 20.03 \mathrm{~N} 170^{\circ} 22.74 \mathrm{E}$ Suiko seamount, Hole $433 \mathrm{~A}, 44^{\circ} 46.60 \mathrm{~N} 170^{\circ} 01.26 \mathrm{E}$ Hole $433 \mathrm{C}, 44^{\circ} 46.63 \mathrm{~N} 170^{\circ} 01.23 \mathrm{E}$

Oceanites, ankaramites, transitional tholeiites, alkali basalts, and hawaiites were recovered from these seamounts. The purpose of this study is the fractionation of trace elements according to their physicochemical properties and partition coefficient classification; fractionation of the elements observed in these samples is compared with their fractionation in mid-oceanic ridge tholeiites. Some preliminary conclusions are presented with regard to mantle heterogeneity or homogeneity and magmatic processes.

\section{DESCRIPTION OF RESULTS}

Ojin Seamount: Hole 430A. Shipboard chemical analyses (major elements and $\mathrm{Ni}, \mathrm{Zr}, \mathrm{Sr}$, and $\mathrm{Ba}$ ) were performed on 13 samples. Major-element data have been completed for $\mathrm{Na}_{2} \mathrm{O}$ (Table 1); trace-element data, obtained either through X-ray fluorescence spectrometry or neutron activation analysis (or both) are shown in Table 2. Shipboard Ba data were obtained without any matrix effect correction; precision should be within 15 per cent, and comparison neutron activation analysis data are given. Except Samples 4-1, 68-70 cm (sandstone) and 6-4, 143-146 cm (tholeiite), all samples have been defined as hawaiites, and show homogeneous results for all elements investigated. Ni and $\mathrm{Cr}$ (high-partition-coefficient elements) concentrations are low (15 and $7 \mathrm{ppm}$, respectively), and low-partition-coefficient elements concentrations are high (e.g., $\mathrm{Nb}=46 \mathrm{ppm}$, $\mathrm{Ta}=3.2 \mathrm{ppm})$.

Nintoku Seamount: Hole $432 A$ (Tables 3 and 4). Sample 1-1, 35-38 cm has been defined as a hawaiite; it shows the highest values for low-partition-coefficient elements (e.g., $\mathrm{Nb}=86 \mathrm{ppm}, \mathrm{Ta}=5.4 \mathrm{ppm}$ ). The five other analyzed samples characterized as alkali basalts also show high concentrations for low-partition-coefficient elements, even if lower than in hawaiites of Hole
430A (e.g., $\mathrm{Nb}=40 \mathrm{ppm}, \mathrm{Ta}=2.7 \mathrm{ppm}$ ). $\mathrm{Ni}$ and $\mathrm{Cr}$ concentrations are higher than in hawaiites (about 60 and $80 \mathrm{ppm}$, respectively).

Suiko Seamount: Holes 433A, 433B, and 433C. The five samples analyzed for Hole 433A (alkali basalts) are very similar both for major elements (Table 5) and trace elements (Table 6), and probably represent different samples of the same flow unit. The sample from Hole 433B is also very close to samples from Hole 433A. According to the concentrations of low-partition-coefficient elements, the samples from Hole 433C (Tables 7 and 8 ) can be classified as follows: oceanites ( $\mathrm{Nb} \sim 5$ $\mathrm{ppm}, \mathrm{Ta} \sim 0.35 \mathrm{ppm})$, ankaramites $(\mathrm{Nb} \sim 11 \mathrm{ppm}, \mathrm{Ta}$ $\sim 0.75 \mathrm{ppm}$ ), transitional tholeiites and (or) transitional alkali basalts $(\mathrm{Nb}=11-17 \mathrm{ppm}, \mathrm{Ta}=0.75-1.1 \mathrm{ppm})$, and one alkali basalt $(\mathrm{Nb}=26.3 \mathrm{ppm}, \mathrm{Ta}=1.79 \mathrm{ppm})$, which is close to alkali basalts of Holes 433A and 433B. Oceanites and ankaramites present high $\mathrm{Ni}$ and $\mathrm{Cr}$ concentrations because of olivine and pyroxene accumulation. From this general description, good agreement is observed between the shipboard classification of samples and low-partition-coefficient element concentrations; in order of increasing concentrations, this classification is: oceanite, ankaramite, "transitional" tholeiites (or "transitional alkali basalts"), alkali basalts, and hawaiites.

\section{Y/Tb, $\mathrm{Zr} / \mathrm{Hf}$, and Nb/Ta RATIOS: CHONDRITIC PRIMORDIAL MANTLE}

From physicochemical properties of the elements and oceanic basalt data, the studied elements have been classified according to their partition coefficients $D_{V}>D_{Y}$ $\cong \mathrm{D}_{\mathrm{Tb}}>\mathrm{D}_{\mathrm{TC}}>\mathrm{D}_{\mathrm{Zr}} \alpha \mathrm{D}_{\mathrm{Hf}}>\mathrm{D}_{\mathrm{Nb}} \cong \mathrm{D}_{\mathrm{Ta}} \neq \mathrm{D}_{\mathrm{La}}>\mathrm{D}_{\mathrm{Th}}$ (Bougault et al., in press). One of the important results is that elements of each pair $\mathrm{Y}-\mathrm{Tb}, \mathrm{Zr}-\mathrm{Hf}$, and $\mathrm{Nb}-\mathrm{Ta}$ belonging to groups III, IV, and V have equal or very similar partition coefficients. These pairs of elements are plotted Figure $1(\mathrm{Y}-\mathrm{Tb})$, Figure 2 (Hf-Zr), and Figure $3(\mathrm{Nb}-\mathrm{Ta})$. We do observe in each diagram that one element of one pair versus the other plots following very closely a straight line. The ratios $\mathrm{Y} / \mathrm{Tb}(40), \mathrm{Zr} / \mathrm{Hf}(40)$, and $\mathrm{Nb} / \mathrm{Ta}(15)$ are very close to those found for the Atlantic Ocean $(42.5,39$, and 17, respectively) (Bougault et al., in press) and to those for the East Pacific Rise (Joron et al., in press). These values correspond to 
TABLE 1

Hole 430A, Ōjin Seamount: Major Elements

\begin{tabular}{|c|c|c|c|c|c|c|c|c|c|c|c|c|c|}
\hline $\begin{array}{l}\text { Sample } \\
\text { (Interval } \\
\text { in } \mathrm{cm} \text { ) }\end{array}$ & $\begin{array}{c}4-1 \\
68-70\end{array}$ & $\begin{array}{c}4-2 \\
139-142\end{array}$ & $\begin{array}{c}5-1(\# 1) \\
5-7\end{array}$ & $\begin{array}{c}5-1(\# 2 A) \\
16-19\end{array}$ & $\begin{array}{c}5-2(\# 6 C) \\
120-123\end{array}$ & $\begin{array}{c}5-5(\# 10 D) \\
127-129\end{array}$ & $\begin{array}{c}\text { 6-1 (\#2B), } \\
33-35\end{array}$ & $\begin{array}{l}\text { 6-2 (\#10) } \\
102-105\end{array}$ & $\begin{array}{c}6-2(\# 13) \\
133-136\end{array}$ & $\begin{array}{c}6-3(\# 6), \\
68-70\end{array}$ & $\begin{array}{c}6-4 \text { (\#1B), } \\
8-11\end{array}$ & $\begin{array}{c}6-4(\# 18) \\
143-146\end{array}$ & $\begin{array}{l}10, C C, \\
28-30\end{array}$ \\
\hline Rock Type & $\begin{array}{l}\text { Volcanic } \\
\text { Sandstone }\end{array}$ & Hawaiite & Hawaiite & Hawaiite & Hawaiite & Hawaiite & Hawaiite & Hawaiite & Hawaiite & Hawaiite & Hawaiite & Tholeiite & Hawaiite \\
\hline $\mathrm{SiO}_{2}$ & 49.77 & 49.03 & 49.96 & 49.10 & 49.50 & 49.81 & 49.60 & 49.29 & 49.80 & 50.02 & 49.56 & 48.38 & 49.63 \\
\hline $\mathrm{TiO}_{2}$ & 2.93 & 3.16 & 3.13 & 3.13 & 3.00 & 3.03 & 3.02 & 2.88 & 2.87 & 2.90 & 2.83 & 2.79 & 2.86 \\
\hline $\mathrm{Al}_{2} \mathrm{O}_{3}$ & 15.16 & 15.96 & 16.09 & 16.17 & 15.57 & 15.84 & 15.72 & 15.99 & 15.98 & 16.05 & 15.75 & 15.44 & 15.76 \\
\hline $\mathrm{Fe}_{2} \mathrm{O}_{3}(\mathrm{~T})$ & 14.04 & 13.02 & 12.41 & 13.28 & 12.87 & 12.22 & 12.31 & 12.39 & 12.00 & 11.72 & 12.35 & 13.07 & 12.46 \\
\hline $\mathrm{MnO}$ & 0.07 & 0.09 & 0.08 & 0.09 & 0.13 & 0.10 & 0.11 & 0.13 & 0.12 & 0.12 & 0.14 & 0.18 & 0.14 \\
\hline MgO & 6.87 & 3.78 & 3.73 & 3.09 & 4.78 & 4.31 & 4.12 & 4.74 & 4.76 & 4.11 & 4.58 & 5.41 & 4.71 \\
\hline $\mathrm{CaO}$ & 3.41 & 6.96 & 7.01 & 7.17 & 6.95 & 7.24 & 7.18 & 6.85 & 7.02 & 7.02 & 6.80 & 11.05 & 6.99 \\
\hline $\mathrm{Na}_{2} \mathrm{O}$ & 4.10 & 4.30 & 4.40 & 4.20 & 4.10 & 4.20 & 4.10 & 4.40 & 4.20 & 4.30 & 4.10 & 2.80 & 4.00 \\
\hline $\mathrm{K}_{2} \mathrm{O}$ & 2.67 & 1.85 & 1.79 & 1.77 & 1.60 & 1.66 & 1.66 & 1.68 & 1.73 & 1.73 & 1.72 & 0.36 & 1.70 \\
\hline $\mathrm{P}_{2} \mathrm{O}_{5}$ & 0.35 & 1.27 & 1.26 & 1.25 & 1.19 & 1.31 & 1.27 & & & & & & 1.29 \\
\hline Total & 99.37 & 99.42 & 99.66 & 99.25 & 99.69 & 99.72 & 99.09 & 99.70 & 99.84 & 99.29 & 99.11 & 99.81 & 99.54 \\
\hline L.O.I. & 25.77 & 4.26 & 4.16 & 4.44 & 3.15 & 3.61 & 3.29 & 3.81 & 3.55 & 3.20 & 3.53 & 1.08 & 2.82 \\
\hline $\mathrm{H}_{2} \mathrm{O}^{+}$ & 2.69 & 1.04 & 0.89 & 0.91 & 1.59 & 1.45 & 1.34 & 1.67 & 1.54 & 1.41 & 1.55 & 0.38 & 1.23 \\
\hline $\mathrm{Co}_{2}$ & 0.15 & 0.15 & 0.04 & 0.18 & 0.03 & 0.05 & 0.07 & 0.07 & 0.06 & 0.07 & 0.05 & 0.03 & 0.05 \\
\hline
\end{tabular}

chondritic values. This means that whatever the magmatic processes giving rise to tholeiites, "transitional" tholeiites, alkali basalts, and hawaiites, and whatever the history of the mantle, these ratios have been kept constant and characterize the primordial chondritic character of the mantle.

\section{Ta-Th, La-Ta: MANTLE SOURCES}

$\mathrm{Nb}, \mathrm{Ta}, \mathrm{Th}$, and La are very low-partition-coefficient elements. $\mathrm{Nb}$ and $\mathrm{Ta}$ physicochemical properties are very similar. $\mathrm{La}, \mathrm{Th}$, and $\mathrm{Ta}$ ionic charges and sizes are different, which leads to different absolute values for their partition coefficients. The consequence is an easier fractionation of these elements compared with the $\mathrm{Nb}$ Ta pair. Two different values have been observed for the $\mathrm{La}$ /Ta ratio $(\sim 9$ and $\sim 18)$, and several values for the $\mathrm{Ta} / \mathrm{Th}$ ratio in oceanic tholeiites. For Leg $55 \mathrm{sam}$ ples (tholeiites, alkali basalts, and oceanites), we did not observe an important fractionation of $\mathrm{Ta} / \mathrm{Th}$ and $\mathrm{La} / \mathrm{Ta}$ (Figures 4 and 5). La/Ta ratios, 9 to 11 for alkali basalts and tholeiites and 12 for oceanites, are in agreement with the value 9 found for tholeiites in the Atlantic samples recovered from topographic highs or "platforms". The almost unique $\mathrm{Ta} / \mathrm{Th}$ ratio for tholeiites and alkali basalts of Leg 55, compared with the possible variation in tholeiites (Bougault et al., in press) and the small difference observed for hawaiites, suggests that the sources of these materials are not much different.

\section{Tb/Ta: PARTIAL MELTING}

Looking at Figure $1(\mathrm{Y}-\mathrm{Tb}$ ), Figure 2 ( $\mathrm{Hf}-\mathrm{Zr}$ ), and Figure $3(\mathrm{Nb}-\mathrm{Ta})$, even if the ratios of the elements of the different pairs are constant or almost constant, the relative positions of tholeiites, alkali basalts of Site 433, alkali basalts of Site 432, and hawaiite of Site 430 are not the same in each diagram. The best classification of these different rock types is made through the lowestpartition-coefficient elements $\mathrm{Nb}-\mathrm{Ta}$ (or $\mathrm{Th}-\mathrm{Ta}$ as well); the tholeiitic sample recovered in Hole 430A (6-4, 143-146 cm) lies in the upper field of the tholeiites; according to the position of the different rock types, only one sample of Hole 433C can be classified as "alkali ba- salt"': 4-1, 39-41 cm; it lies very close to alkali basalts of Holes 433A and 433B.

With increasing partition coefficients from $\mathrm{Nb}-\mathrm{Ta}$ to $\mathrm{Hf}-\mathrm{Zr}$ to $\mathrm{Y}-\mathrm{Tb}$, alkali basalts plot closer and closer to the tholeiitic field; alkali basalts of Site 432 plot in the middle part of the tholeiitic field (with some fractionation) in the $\mathrm{Y}-\mathrm{Tb}$ diagram (Figure 1).

This feature is easier to see in Figure 6, where two elements of the same transition series (with different partition coefficients) are plotted (Tb-Ta). If we admit from $\mathrm{La} / \mathrm{Ta}$ and $\mathrm{Th} / \mathrm{Ta}$ data that the mantle sources, parents of these different rocks, are very similar, then the only way to explain such $\mathrm{Tb} / \mathrm{Ta}$ fractionation is partial melting. It can be the extent of partial melting: in this case, except for the hawaiite sample of Hole 432A, it can be stated that the lowest partial melting is related to alkali basalts of Hole 432A. It can also be melting of a residue; in this second possibility, tholeiites (or transitional tholeiites) would be the result of melting of a residue, alkali basalts being produced through melting a more primitive source. Both hypotheses, related to partial melting, can account for the relative values of $\mathrm{Tb} / \mathrm{Ta}$ ratios in tholeiites and alkali basalts.

\section{V, Ti: FRACTIONAL CRYSTALLIZATION}

According to their partition coefficients, $\mathrm{V}$ and $\mathrm{Ti}$ are the closest to $\mathrm{Y}$ and $\mathrm{Zr}$, respectively. In respect of "nonfractionation" availability, $\mathrm{V}-\mathrm{Y}$ and $\mathrm{Ti}-\mathrm{Zr}$ are plotted in Figures 7 and 8 . We indeed observe very little fractionation between the elements of these pairs for tholeiites and alkali basalts. But for hawaiites, a large fractionation is observed, these two diagrams showing the biggest difference between hawaiites and alkali or tholeiite basalts. This fractionation is interpreted as the result of titanomagnetite fractionation (see petrographic description in this volume).

\section{CONCLUSION}

According to the classification of the elements which was made previously from the samples recovered in the Atlantic Ocean, with regard to their physicochemical 


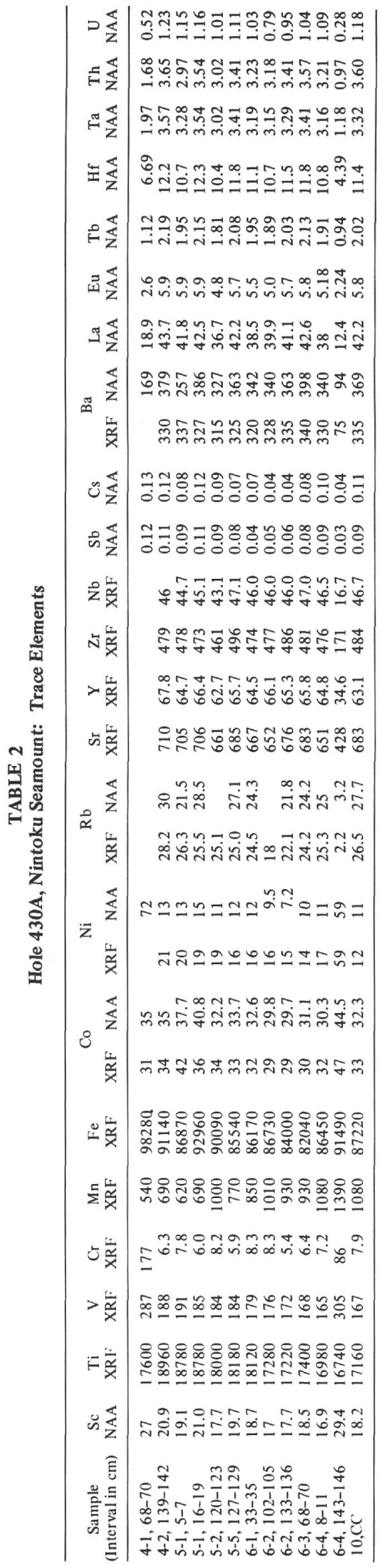

TABLE 3

Hole 432A, Nintoku Seamount: Major Elements

\begin{tabular}{|c|c|c|c|c|c|c|}
\hline $\begin{array}{l}\text { Sample } \\
\text { (Interval } \\
\text { in } \mathrm{cm} \text { ) }\end{array}$ & $\begin{array}{c}1-1 \\
35-38\end{array}$ & $\begin{array}{c}2-1(\# 8 B), \\
83-85\end{array}$ & $\begin{array}{c}2-2(\# 11 E) \\
100-102\end{array}$ & $\begin{array}{c}3-2 \\
106-108\end{array}$ & $\begin{array}{c}4-4 \text { (\#6B), } \\
84-86\end{array}$ & $\begin{array}{c}5-1(\# 3 B), \\
128-130\end{array}$ \\
\hline $\begin{array}{l}\text { Rock } \\
\text { Type }\end{array}$ & Hawaiite & $\begin{array}{l}\text { Alkali } \\
\text { Basalt }\end{array}$ & $\begin{array}{l}\text { Alkali } \\
\text { Basalt }\end{array}$ & $\begin{array}{l}\text { Alkali } \\
\text { Basalt }\end{array}$ & $\begin{array}{l}\text { Alkali } \\
\text { Basalt }\end{array}$ & $\begin{array}{l}\text { Alkali } \\
\text { Basalt }\end{array}$ \\
\hline $\mathrm{SiO}_{2}$ & 48.35 & 46.75 & 47.49 & 46.64 & 47.91 & 47.69 \\
\hline $\mathrm{TiO}_{2}^{2}$ & 2.84 & 2.65 & 2.51 & 3.01 & 3.38 & 3.01 \\
\hline $\mathrm{Al}_{2} \mathrm{O}_{3}$ & 20.38 & 16.35 & 18.38 & 15.54 & 17.40 & 15.72 \\
\hline $\mathrm{Fe}_{2} \mathrm{O}_{3}(\mathrm{~T})$ & 10.95 & 13.19 & 12.78 & 14.58 & 15.58 & 14.47 \\
\hline $\mathrm{MnO}$ & 0.07 & 0.16 & 0.12 & 0.19 & 0.27 & 0.17 \\
\hline $\mathrm{MgO}$ & 2.13 & 6.21 & 3.10 & 5.79 & 3.94 & 5.52 \\
\hline $\mathrm{CaO}$ & 7.39 & 10.19 & 9.97 & 8.36 & 5.87 & 8.30 \\
\hline $\mathrm{Na}_{2} \mathrm{O}$ & 4.70 & 3.10 & 3.40 & 3.50 & 3.30 & 3.30 \\
\hline $\mathrm{K}_{2} \mathrm{O}$ & 1.64 & 0.95 & 1.28 & 1.19 & 1.41 & 1.09 \\
\hline $\mathrm{P}_{2} \mathrm{O}_{5}$ & 1.12 & 0.52 & 0.58 & 0.49 & 0.54 & 0.48 \\
\hline Total & 99.59 & 100.08 & 99.62 & 99.29 & 99.59 & 99.75 \\
\hline L.O.I. & 7.15 & 4.14 & 4.62 & 1.38 & 7.79 & 2.7 \\
\hline $\mathrm{H}_{2} \mathrm{O}^{+}$ & 2.66 & 1.97 & 1.63 & 2.62 & 3.15 & 1.85 \\
\hline $\mathrm{CO}_{2}$ & 0.46 & 0.29 & 0.31 & 0.48 & 0.07 & 0.10 \\
\hline
\end{tabular}

properties and partition coefficients, the following items can be formulated from the study of the Emperor Seamount samples drilled during Leg 55:

1) The chondritic nature of the "primordial" mantle is confirmed through $\mathrm{Y} / \mathrm{Tb}, \mathrm{Zr} / \mathrm{Hf}$, and $\mathrm{Nb} / \mathrm{Ta}$ ratios.

2) The value 9 for the $\mathrm{La} / \mathrm{Ta}$ ratio has been found related to topographic highs or platforms in the Atlantic Ocean (the value is 18 in other cases, corresponding to typically "depleted" tholeiites). The values close to 9 found for Emperor Seamount samples independently of their petrographic classification (tholeiite, alkali basalts, or hawaiite) tend to confirm the correlation of this ratio to topography.

3) The single $\mathrm{Th} / \mathrm{Ta}$ ratio observed in tholeiites, alkali basalts, and hawaiites suggests similar mantle sources for these different rock types.

4) The transition elements belonging to the same series, characterized by different partition coefficients, show a fractionation. This fractionation can be interpreted as the result of partial melting.

5) $\mathrm{Ti}$ and $\mathrm{V}$ are fractionated in hawaiites as a consequence of titanomagnetite crystallization.

\section{REFERENCES}

Bougault, H., Treuil, M., and Joron, J. L., in press. Trace elements in basalts from $22^{\circ} \mathrm{N}$ and $36^{\circ} \mathrm{N}$ in the Atlantic Ocean: Fractional crystallization, partial melting, and heterogeneity of the upper mantle. In Melson, W. G., Rabinowitz, P., et al., Initial Reports of the Deep Sea Drilling Project, v. 45: Washington (U. S. Government Printing Office), pp. 493-506.

Joron, J. L., Bollinger, C., Quisefit, J. P., Bougault, H., and Treuil, M., in press. Trace elements in basalts at $25^{\circ} \mathrm{N}$, old crust, in the Atlantic Ocean: alteration, mantle and magmatic processes. In Donnelly, T. and Francheteau, J., Bryan, W., Robinson, P., Flower, M., Salisbury, M., Initial Reports of the Deep Sea Drilling Project, v. 51, 52, 53, Part 2: Washington (U.S. Government Printing Office).

Bougault, H., Joron, J. L., and Treuil, M., in press. Alteration, fractional crystallization, partial melting, mantle properties from trace elements recovered in the North Atlantic. Maurice Ewing Series, American Geophysical Union. 
TABLE 4

Hole 432A, Nintoku Seamount: Trace Elements

\begin{tabular}{|c|c|c|c|c|c|c|c|c|c|c|c|c|c|c|c|c|}
\hline \multirow{2}{*}{$\begin{array}{l}\text { Sample } \\
\text { (Interval in cm) }\end{array}$} & \multirow{2}{*}{$\begin{array}{l}\text { Sc } \\
\text { NAA }\end{array}$} & \multirow{2}{*}{$\begin{array}{c}\mathrm{Ti} \\
\mathrm{XRF}\end{array}$} & \multirow{2}{*}{$\begin{array}{c}\mathrm{V} \\
\mathrm{XRF}\end{array}$} & \multirow{2}{*}{$\begin{array}{c}\mathrm{Cr} \\
\mathrm{XRF}\end{array}$} & \multirow{2}{*}{$\begin{array}{c}\mathrm{Mn} \\
\mathrm{XRF}\end{array}$} & \multirow{2}{*}{$\begin{array}{c}\dot{\mathrm{Fe}} \\
\mathrm{XRF}\end{array}$} & \multicolumn{2}{|c|}{ Co } & \multicolumn{2}{|c|}{$\mathrm{Ni}$} & \multicolumn{2}{|c|}{$\mathrm{Rb}$} & \multirow{2}{*}{$\underset{\mathrm{XRF}}{\mathrm{Y}}$} & \multirow{2}{*}{$\underset{\mathrm{Zr}}{\mathrm{ZrF}}$} & \multirow{2}{*}{$\begin{array}{c}\mathrm{Nb} \\
\mathrm{XRF}\end{array}$} & \multirow{2}{*}{$\begin{array}{c}\mathrm{Sb} \\
\mathrm{NAA}\end{array}$} \\
\hline & & & & & & & XRF & NAA & XRF & NAA & XRF & NAA & & & & \\
\hline $1-1,35-38$ & 13.6 & 17040 & 117 & 10.4 & 540 & 76650 & 45 & 45.8 & 20 & 14 & 13.2 & 10 & 40.6 & 303 & 86.5 & 0.18 \\
\hline $2-1,83-85$ & 22.1 & 15900 & 248 & 92.8 & 1240 & 92330 & 45 & 44.1 & 81 & 75 & 14.4 & 14.5 & 23 & 175 & 35.1 & 0.05 \\
\hline $2-2,100-102$ & 20 & 15060 & 233 & 79.6 & 330 & 89460 & 39 & 37.4 & 70 & 72 & 20 & 18.3 & 25.9 & 181 & 37.2 & 0.05 \\
\hline $3-2,106-108$ & 21.8 & 18060 & 223 & 82.6 & 1470 & 102060 & 41 & 40 & 51 & 51 & 20.3 & & 30.1 & 204 & 40.0 & 0.04 \\
\hline \multirow{19}{*}{$\begin{array}{l}4-4,84-86 \\
5-1,128-130\end{array}$} & 24.3 & 20280 & 247 & 91.4 & 2090 & 109060 & 45 & 43.8 & 62 & 56 & 22 & 22 & 31.9 & 226 & 41.0 & 0.02 \\
\hline & 22.4 & 18060 & 220 & 79.0 & 1316 & 101290 & 41 & 39.1 & 53 & 47 & 16.8 & 17.7 & 30.5 & 194 & 37.9 & 0.02 \\
\hline & & & & & & & \multicolumn{10}{|c|}{$\begin{array}{c}\text { TABLE } 5 \\
\text { Holes 433A and 433B, Suiko Seamount: Major Elements }\end{array}$} \\
\hline & & & & & & & & $\begin{array}{l}\text { imple } \\
\text { nterval } \\
c m\end{array}$ & \multicolumn{2}{|c|}{$\begin{array}{c}433 \mathrm{~A}-20-1 \\
\quad(11 \mathrm{~d}), \\
36-38\end{array}$} & $\begin{array}{l}433 \mathrm{~A}-20-2 \\
(\# 1 \mathrm{~b}) \\
10-12\end{array}$ & $\begin{array}{c}433 \mathrm{~A}-20-2 \\
(\# 2 \mathrm{e}) \\
49-51\end{array}$ & $\begin{array}{c}433 \mathrm{~A}-21-2 \\
(\# 2 \mathrm{c}) \\
84\end{array}$ & \multicolumn{2}{|c|}{$\begin{array}{cc}233 A-21-4 \\
(\# 15) \\
138-140\end{array}$} & $\begin{array}{l}433 \mathrm{~B}-5-2 \\
(\# 3-2) \\
81-83\end{array}$ \\
\hline & & & & & & & & ock & \multirow{2}{*}{\multicolumn{2}{|c|}{$\begin{array}{l}\text { Alkali } \\
\text { Basalt }\end{array}$}} & Alkali & \multirow{2}{*}{$\begin{array}{l}\text { Alkali } \\
\text { Basalt }\end{array}$} & Alkali & \multirow{2}{*}{\multicolumn{2}{|c|}{$\begin{array}{l}\text { Alkali } \\
\text { Basalt }\end{array}$}} & Alkali \\
\hline & & & & & & & & ype & & & Basalt & & Basalt & & & Basalt \\
\hline & & & & & & & & $\mathrm{O}_{2}$ & \multicolumn{2}{|c|}{47.18} & 47.08 & 46.93 & 47.32 & \multicolumn{2}{|c|}{47.70} & 47.46 \\
\hline & & & & & & & & & 3.1 & & 3.33 & \multirow{2}{*}{$\begin{array}{r}3.17 \\
13.91\end{array}$} & 3.19 & & 3.21 & 3.18 \\
\hline & & & & & & & & ${ }_{2} \mathrm{O}_{3}$ & 14.3 & & 14.82 & & 14.29 & & 4.55 & 14.42 \\
\hline & & & & & & & & ${ }_{2} \mathrm{O}_{3} \mathrm{O}(\mathrm{T})$ & 15.1 & & 15.40 & 15.49 & 15.05 & & 4.96 & 14.98 \\
\hline & & & & & & & & & .18 & & $\begin{array}{r}.16 \\
4.60\end{array}$ & $\begin{array}{r}.21 \\
5.39\end{array}$ & $\begin{array}{r}.19 \\
5.57\end{array}$ & & $\begin{array}{r}.18 \\
507\end{array}$ & $\begin{array}{l}0.18 \\
5.45\end{array}$ \\
\hline & & & & & & & & & 0.5 & & $\begin{array}{l}4.60 \\
8.92\end{array}$ & $\begin{array}{l}5.39 \\
9.77\end{array}$ & 9.31 & & $\begin{array}{l}5.07 \\
936\end{array}$ & $\begin{array}{l}3.45 \\
9.88\end{array}$ \\
\hline & & & & & & & & ${ }_{2} \mathrm{O}$ & $\begin{array}{l}9.3 \\
3.3\end{array}$ & & $\begin{array}{l}8.92 \\
3.40\end{array}$ & 3.10 & $\begin{array}{l}9.21 \\
3.20\end{array}$ & & $\begin{array}{l}5.30 \\
3.20\end{array}$ & $\begin{array}{l}3.00 \\
3.10\end{array}$ \\
\hline & & & & & & & & $2 \mathrm{O}$ & .9 & & 1.07 & 1.03 & .93 & & .97 & 0.86 \\
\hline & & & & & & & & $2_{5}$ & 4 & 6 & .44 & .48 & .46 & & .45 & 0.44 \\
\hline & & & & & & & & otal & 99.3 & & 99.22 & 99.47 & 99.41 & & 9.65 & 99.95 \\
\hline & & & & & & & & O.I. & 1.6 & & 2.93 & 3.16 & 2.45 & & 2.27 & 1.27 \\
\hline & & & & & & & & $2 \mathrm{O}^{+}$ & .7 & & .93 & .95 & 1.10 & & .99 & 0.72 \\
\hline & & & & & & & & $\mathrm{O}_{2}$ & & & .29 & .83 & .10 & & .09 & 0.42 \\
\hline
\end{tabular}

TABLE 6

Holes 433A and 433B, Suiko Seamount: Trace Elements

\begin{tabular}{|c|c|c|c|c|c|c|c|c|c|c|c|c|c|c|c|c|c|c|c|c|c|c|c|c|c|c|c|}
\hline \multirow{2}{*}{$\begin{array}{c}\text { Sample } \\
\text { (Interval in } \mathrm{cm} \text { ) }\end{array}$} & \multirow{2}{*}{$\begin{array}{l}\mathrm{Sc} \\
\text { NAA }\end{array}$} & \multirow{2}{*}{$\begin{array}{c}\mathrm{Ti} \\
\mathrm{XRF}\end{array}$} & \multirow{2}{*}{$\begin{array}{c}\mathrm{V} \\
\mathrm{XRF}\end{array}$} & \multirow{2}{*}{$\begin{array}{c}\mathrm{Cr} \\
\mathrm{XRF}\end{array}$} & \multirow{2}{*}{$\begin{array}{l}\text { Mn } \\
\text { XRF }\end{array}$} & \multirow{2}{*}{$\begin{array}{c}\mathrm{Fe} \\
\mathrm{XRF}\end{array}$} & \multicolumn{2}{|c|}{ Co } & \multicolumn{2}{|c|}{$\mathrm{Ni}$} & \multicolumn{2}{|c|}{$\mathrm{Rb}$} & \multirow{2}{*}{$\underset{\mathrm{XRF}}{\mathrm{Sr}}$} & \multirow{2}{*}{$\begin{array}{c}\mathrm{Y} \\
\mathrm{XRF}\end{array}$} & \multirow{2}{*}{$\begin{array}{c}\mathrm{Zr} \\
\mathrm{XRF}\end{array}$} & \multirow{2}{*}{$\begin{array}{l}\mathrm{Nb} \\
\mathrm{XRF}\end{array}$} & \multirow{2}{*}{$\begin{array}{c}\text { Sb } \\
\text { NAA }\end{array}$} & \multirow{2}{*}{$\begin{array}{c}\mathrm{Cs} \\
\text { NAA }\end{array}$} & \multicolumn{2}{|c|}{$\mathrm{Ba}$} & \multirow{2}{*}{$\begin{array}{c}\mathrm{La} \\
\text { NAA }\end{array}$} & \multirow{2}{*}{$\begin{array}{c}\text { Eu } \\
\text { NAA }\end{array}$} & \multirow{2}{*}{$\begin{array}{c}\mathrm{Tb} \\
\text { NAA }\end{array}$} & \multirow{2}{*}{$\begin{array}{c}\mathrm{Hf} \\
\text { NAA }\end{array}$} & \multirow{2}{*}{$\begin{array}{c}\mathrm{Ta} \\
\text { NAA }\end{array}$} & \multirow{2}{*}{$\begin{array}{c}\text { Th } \\
\text { NAA }\end{array}$} & \multirow{2}{*}{$\begin{array}{r}\text { U } \\
\text { NAA }\end{array}$} \\
\hline & & & & & & & XRF & NAA & $\mathrm{XRF}$ & NAA & XRF & NAA & & & & & & & XRF & NAA & & & & & & & \\
\hline \multicolumn{28}{|l|}{ Hole 433A } \\
\hline $20-1,36-38$ & 31.4 & 18960 & 365 & 44.7 & 1390 & 106120 & 46 & 43.6 & 49 & 44 & 14.4 & & 404 & 40.1 & 217 & 28.5 & 0.03 & 0.06 & 171 & 220 & 21.2 & 2.49 & 1.03 & 5.44 & 2.01 & 1.86 & 0.50 \\
\hline $20-2,10-12$ & 32.2 & 19980 & 385 & 44.4 & 1240 & 107800 & 46 & 44.8 & 53 & 46 & 15.3 & & 420 & 38.3 & 223 & 28.8 & 0.05 & 0.11 & 180 & 224 & 20.5 & 2.58 & 1.02 & 5.33 & 2.01 & 1.89 & 0.48 \\
\hline $20-2,49-51$ & 30.6 & 19020 & 357 & 43.3 & 1626 & 108360 & 47 & 44 & 50 & 46 & 20.6 & 19.5 & 391 & 39.7 & 212 & 29.1 & 0.05 & 0.97 & 170 & 202 & 19.6 & 2.40 & 1.04 & 5.1 & 1.99 & 1.86 & 0.55 \\
\hline $21-2,84-86$ & 32 & 19140 & 362 & 43.0 & 1470 & 105350 & 47 & 45.1 & 48 & 48 & 14 & 12.8 & 384 & 38.3 & 218 & 28.7 & 0.02 & 0.06 & 165 & 199 & 20 & 2.59 & 1.01 & 5.42 & 2.02 & 1.94 & 0.56 \\
\hline $21-4,138-140$ & 31.7 & 19260 & 364 & 40.5 & 1390 & 104720 & 46 & 43 & 50 & 43 & 14.8 & 13.4 & 409 & 38.1 & 221 & 28.3 & 0.01 & 0.01 & 181 & 228 & 21 & 2.58 & 1.03 & 5.52 & 2.05 & 1.93 & 0.40 \\
\hline \multicolumn{28}{|l|}{ Hole 433B } \\
\hline $5-2,81-83$ & 32.8 & 19080 & 370 & 51.5 & 1390 & 104860 & 47 & 46.6 & 50 & 50 & 13.3 & 12.2 & 411 & 37.1 & 217 & 28.5 & 0.02 & 0.06 & 173 & 203 & 20.1 & 2.49 & 0.98 & 5.27 & 1.98 & 1.87 & 0.40 \\
\hline
\end{tabular}


TABLE 7

Hole 433C, Suiko Seamount: Major Elements

\begin{tabular}{|c|c|c|c|c|c|c|c|c|c|c|c|}
\hline $\begin{array}{l}\text { Sample } \\
\text { (Interval } \\
\text { in } \mathrm{cm} \text { ) }\end{array}$ & $\begin{array}{c}\text { 4-1 (\#2C), } \\
39-41\end{array}$ & $\begin{array}{c}10-1(\# 1 A), \\
7-9\end{array}$ & $\begin{array}{c}10-2(\# 3 A), \\
16-18\end{array}$ & $\begin{array}{c}10-2(\# 7) \\
58-60\end{array}$ & $\begin{array}{c}10-3(\# 10 A) \\
105-107\end{array}$ & $\begin{array}{c}10-4 \text { (\#8B), } \\
118-121\end{array}$ & $\begin{array}{c}10-5(\# 13) \\
118-120\end{array}$ & $\begin{array}{c}11-1(\# 9 C), \\
135-137\end{array}$ & $\begin{array}{c}11-3(\# 2 A) \\
29-31\end{array}$ & $\begin{array}{c}11-4(\# 14), \\
110-112\end{array}$ & $\begin{array}{c}12-3(\# 1 \mathrm{E}), \\
51-53\end{array}$ \\
\hline Rock Type & Alkali Basalt & Ankaramite & Ankaramite & Ankaramite & Ankaramite & Ankaramite & Tholeiite & Tholeiite & Tholeiite & $\begin{array}{c}\text { Alkali Basalt } \\
\text { (Transitional) }\end{array}$ & Tholeiite \\
\hline $\mathrm{SiO}_{2}$ & 46.99 & 46.86 & 46.70 & 46.16 & 45.96 & 45.94 & 47.78 & 47.00 & 48.00 & 48.73 & 48.91 \\
\hline $\mathrm{TiO}_{2}$ & 2.92 & 1.70 & 1.85 & 1.91 & 1.97 & 1.15 & 1.89 & 1.89 & 1.92 & 2.40 & 2.35 \\
\hline $\mathrm{Al}_{2} \mathrm{O}_{3}$ & 13.73 & 12.74 & 13.09 & 14.10 & 14.76 & 8.81 & 14.14 & 14.86 & 15.00 & 15.33 & 14.36 \\
\hline $\mathrm{l}_{2} \mathrm{e}_{2} \mathrm{O}_{3}(\mathrm{~T})$ & 14.93 & 14.15 & 14.16 & 14.63 & 14.95 & 14.10 & 15.70 & 13.35 & 13.03 & 12.71 & 12.30 \\
\hline $\mathrm{MnO}$ & 0.19 & 0.16 & 0.15 & 0.13 & 0.15 & 0.19 & 0.07 & 0.18 & 0.12 & 0.11 & 0.14 \\
\hline $\mathrm{MgO}$ & 7.39 & 14.05 & 14.09 & 11.34 & 9.23 & 20.39 & 10.81 & 8.02 & 8.25 & 6.95 & 7.43 \\
\hline $\mathrm{CaO}$ & 9.72 & 7.71 & 6.54 & 7.84 & 8.95 & 6.70 & 4.82 & 10.95 & 10.54 & 10.25 & 11.30 \\
\hline $\mathrm{Na}_{2} \mathrm{O}$ & 2.80 & 1.90 & 2.00 & 2.30 & 2.20 & 1.40 & 2.80 & 2.50 & 2.50 & 2.50 & 2.50 \\
\hline $\mathrm{K}_{2} \mathrm{O}$ & 0.79 & 0.40 & 0.47 & 0.44 & 0.71 & 0.28 & 0.83 & 0.32 & 0.23 & 0.63 & 0.20 \\
\hline $\mathrm{P}_{2} \mathrm{O}_{5}$ & 0.40 & 0.25 & 0.21 & 0.24 & 0.25 & 0.15 & 0.23 & 0.24 & 0.22 & 0.28 & 0.28 \\
\hline Total & 99.86 & 99.92 & 99.26 & 99.09 & 99.13 & 99.11 & 99.07 & 99.31 & 99.81 & 99.89 & 99.77 \\
\hline L.O.I. & 1.28 & 6.49 & 10.40 & 6.59 & 7.83 & 14.00 & 8.74 & 7.24 & 5.41 & 5.66 & 3.38 \\
\hline $\mathrm{H}_{2} \mathrm{O}^{+}$ & 1.12 & 4.17 & 4.62 & 3.56 & 2.98 & 5.52 & 3.26 & 2.28 & 2.19 & 1.72 & 1.12 \\
\hline $\mathrm{CO}_{2}$ & 0.05 & 0.20 & 0.20 & 0.17 & 0.37 & 1.76 & 0.17 & 1.31 & 0.51 & 0.56 & 0.47 \\
\hline
\end{tabular}

TABLE 7 - Continued

\begin{tabular}{|c|c|c|c|c|c|c|c|c|c|c|c|}
\hline $\begin{array}{l}\text { Sample } \\
\text { (Interval } \\
\text { in } \mathrm{cm} \text { ) }\end{array}$ & $\begin{array}{c}13-2(\# 1 \mathrm{H}), \\
53-55\end{array}$ & $\begin{array}{c}14-3(\# 4 A), \\
55-57\end{array}$ & $\begin{array}{c}14-4(\# 1 B), \\
21-23\end{array}$ & $\begin{array}{c}15-1(\# 13) \\
115-118\end{array}$ & $\begin{array}{c}15-4(\# 4), \\
46-48\end{array}$ & $\begin{array}{c}15-6(\# 1 C) \\
31-32\end{array}$ & $\begin{array}{c}19-2(\# 2 F), \\
56-58\end{array}$ & $\begin{array}{c}19-4(\# 1 B), \\
17-19\end{array}$ & $\begin{array}{c}20-2(\# 13) \\
132-134\end{array}$ & $\begin{array}{c}21-4 \\
40-42\end{array}$ & $\begin{array}{c}22-1(\# 10), \\
94-96\end{array}$ \\
\hline Rock Type & Tholeiite & $\begin{array}{l}\text { Alkali Basalt } \\
\text { (Transitional) }\end{array}$ & Tholeiite & Tholeiite & Tholeiite & Tholeiite & Tholeiite & Tholeiite & Tholeiite & $\begin{array}{c}\text { Tholeiite } \\
\text { (Plag. Phyric) }\end{array}$ & $\begin{array}{c}\text { Alkali Basalt } \\
\text { (Transitional) }\end{array}$ \\
\hline $\mathrm{SiO}_{2}$ & 48.00 & 48.26 & 48.62 & 47.78 & 47.62 & 47.91 & 48.31 & 48.83 & 48.24 & 47.26 & 47.47 \\
\hline $\mathrm{TiO}_{2}^{2}$ & 2.26 & 2.68 & 2.53 & 2.65 & 2.54 & 2.57 & 2.45 & 2.10 & 2.83 & 2.71 & 2.77 \\
\hline $\mathrm{Al}_{2} \mathrm{O}_{3}$ & 14.22 & 15.50 & 15.23 & 14.75 & 13.39 & 13.87 & 14.93 & 15.13 & 15.80 & 15.73 & 15.24 \\
\hline $\mathrm{Fe}_{2} \mathrm{O}_{3}(\mathrm{~T})$ & 13.16 & 13.97 & 13.55 & 14.32 & 16.88 & 14.50 & 13.26 & 11.98 & 12.32 & 12.92 & 13.31 \\
\hline $\mathrm{MgO}$ & 7.90 & 7.20 & 7.00 & 7.35 & 8.30 & 6.50 & 7.33 & 7.70 & 7.25 & 7.58 & 6.96 \\
\hline $\mathrm{CaO}$ & 11.16 & 7.39 & 9.63 & 8.84 & 6.59 & 10.77 & 10.22 & 10.72 & 9.50 & 9.16 & 9.21 \\
\hline $\mathrm{Na}_{2} \mathrm{O}$ & 2.30 & 3.20 & 2.90 & 2.90 & 2.80 & 2.50 & 2.70 & 2.70 & 2.90 & 3.00 & 2.90 \\
\hline $\mathrm{K}_{2}{ }_{2} \mathrm{O}$ & 0.47 & 0.80 & 0.22 & 0.46 & 1.24 & 0.24 & 0.30 & 0.24 & 0.30 & 0.45 & 0.64 \\
\hline $\mathrm{P}_{2} \mathrm{O}_{5}$ & & & & 0.33 & 0.24 & 0.28 & 0.27 & 0.26 & 0.29 & 0.29 & 0.31 \\
\hline Total & 99.92 & 99.40 & 100.12 & 99.50 & 99.71 & 99.32 & 99.90 & 99.79 & 99.54 & 99.21 & 98.97 \\
\hline L.O.I. & 2.47 & 4.64 & 3.37 & 3.28 & 6.56 & 1.33 & 2.78 & 2.86 & 3.57 & 3.67 & 3.52 \\
\hline $\mathrm{H}_{2} \mathrm{O}^{+}$ & 1.42 & 1.93 & 1.13 & 1.37 & 1.97 & 0.42 & 1.21 & 1.23 & 1.50 & 1.41 & 1.70 \\
\hline $\mathrm{CO}_{2}$ & 0.14 & 0.26 & 0.19 & 0.17 & 0.12 & 0.09 & 0.28 & 0.24 & 0.30 & 0.27 & 0.30 \\
\hline
\end{tabular}

TABLE 7 - Continued

\begin{tabular}{|c|c|c|c|c|c|c|c|c|c|c|c|}
\hline $\begin{array}{l}\text { Sample } \\
\text { (Interval } \\
\text { in } \mathrm{cm} \text { ) }\end{array}$ & $\begin{array}{c}24-1(\# 2), \\
12-15\end{array}$ & $\begin{array}{c}23-1(\# 5 D) \text {, } \\
102-104\end{array}$ & $\begin{array}{c}23-5(\# 1 B) \\
16-19\end{array}$ & $\begin{array}{c}24-7(\# 3 G), \\
141-144\end{array}$ & $\begin{array}{c}25-2(\# 1 B), \\
22-25\end{array}$ & $\begin{array}{c}26-1(\# 2 A), \\
42-45\end{array}$ & $\begin{array}{c}26-5(\# 1 \mathrm{H}), \\
82-85\end{array}$ & $\begin{array}{c}26-6(\# 7 A), \\
114-117\end{array}$ & $\begin{array}{c}27-2(\# 6 B), \\
131-134\end{array}$ & $\begin{array}{c}28-2(\# 1 D), \\
19-21\end{array}$ & $\begin{array}{c}28-5 \text { (\#6E) } \\
107-109\end{array}$ \\
\hline Rock Type & $\begin{array}{l}\text { Tholeiite } \\
\text { (Oceanite) }\end{array}$ & Tholeiite & Tholeiite & $\begin{array}{l}\text { Tholeiite } \\
\text { (Oceanite) }\end{array}$ & Tholeiite & Tholeiite & Tholeiite & Tholeiite & $\begin{array}{l}\text { Tholeiite } \\
\text { (Oceanite) }\end{array}$ & Tholeiite & Tholeiite \\
\hline $\mathrm{SiO}_{2}$ & 46.10 & 48.45 & 49.11 & 44.73 & 47.40 & 47.88 & 46.94 & 46.65 & 44.00 & 49.00 & 49.35 \\
\hline $\mathrm{TiO}_{2}$ & 1.33 & 2.32 & 1.69 & 1.24 & 2.10 & 2.10 & 2.10 & 2.37 & 1.08 & 2.57 & 2.78 \\
\hline $\mathrm{Al}_{2} \mathrm{O}_{3}$ & 9.76 & 13.27 & 12.84 & 8.88 & 14.71 & 14.70 & 13.04 & 14.75 & 8.04 & 13.97 & 14.11 \\
\hline $\mathrm{Fe}_{2} \mathrm{O}_{3}(\mathrm{~T})$ & 14.24 & 16.00 & 12.31 & 14.04 & 12.98 & 12.98 & 13.67 & 13.32 & 14.67 & 13.24 & 13.71 \\
\hline $\mathrm{MnO}$ & 0.18 & 0.08 & 0.15 & 0.17 & 0.14 & 0.14 & 0.16 & 0.15 & 0.18 & 0.16 & 0.16 \\
\hline $\mathrm{MgO}$ & 19.60 & 9.61 & 12.54 & 22.00 & 9.56 & 8.29 & 12.66 & 10.88 & 26.02 & 6.89 & 6.52 \\
\hline $\mathrm{CaO}$ & 6.25 & 5.01 & 8.03 & 5.33 & 9.67 & 10.30 & 8.98 & 8.47 & 4.29 & 10.29 & 10.61 \\
\hline $\mathrm{Na}_{2} \mathrm{O}$ & 1.20 & 2.60 & 2.20 & 1.60 & 2.40 & 2.50 & 2.00 & 2.50 & 1.00 & 2.50 & 2.60 \\
\hline $\mathrm{K}_{2} \mathrm{O}$ & 0.13 & 1.08 & 0.27 & 1.08 & 0.16 & 0.22 & 0.14 & 0.14 & 0.13 & 0.19 & 0.21 \\
\hline $\mathrm{P}_{2} \mathrm{O}_{5}$ & 0.13 & 0.06 & 0.18 & $\begin{array}{l}1.00 \\
0.14\end{array}$ & 0.21 & 0.23 & $\begin{array}{l}0.14 \\
0.23\end{array}$ & 0.24 & 0.11 & 0.25 & 0.33 \\
\hline Total & 98.92 & 98.48 & 99.32 & 99.11 & 99.33 & 99.34 & 99.92 & 99.47 & 99.52 & 99.06 & 100.38 \\
\hline L.O.I. & 6.10 & 8.54 & 6.33 & 4.31 & 4.12 & 3.09 & 4.82 & 3.14 & 5.89 & 1.11 & 1.44 \\
\hline $\mathrm{H}_{2} \mathrm{O}^{+}$ & 4.89 & 3.28 & 2.86 & 3.36 & 2.27 & 1.48 & 3.47 & 3.11 & 4.46 & 0.59 & 0.59 \\
\hline $\mathrm{CO}_{2}$ & 0.52 & 0.25 & 0.47 & 0.24 & 0.20 & 0.18 & 0.36 & 0.38 & 0.34 & 0.17 & 0.10 \\
\hline
\end{tabular}


TABLE 7 - Continued

\begin{tabular}{|c|c|c|c|c|c|c|c|c|c|c|c|}
\hline $\begin{array}{l}\text { Sample } \\
\text { (Interval } \\
\text { in } \mathrm{cm} \text { ) }\end{array}$ & $\begin{array}{c}29-3(\# 2 C), \\
25-27\end{array}$ & $\begin{array}{c}31-1 \text { (\#1D), } \\
15-18\end{array}$ & $\begin{array}{l}32-1(\# 3 D), \\
98-101\end{array}$ & $\begin{array}{c}32-5(\# 1 C), \\
72-75\end{array}$ & $\begin{array}{l}34-7 \\
95-98\end{array}$ & $\begin{array}{c}35-6 \text { (\#1D), } \\
27-29\end{array}$ & $\begin{array}{c}\text { 36-2 (\#1D), } \\
\text { 16-19 }\end{array}$ & $\begin{array}{c}36-3(\# 1 \mathrm{~J}), \\
79-82\end{array}$ & $\begin{array}{c}36-4(\# 1 F), \\
49-52\end{array}$ & $\begin{array}{c}36-5(\# 4 M), \\
119-122\end{array}$ & $\begin{array}{c}37-3 \text { (\#1F), } \\
101-103\end{array}$ \\
\hline Rock Type & $\begin{array}{c}\text { Tholeiite } \\
\text { (Transitional) }\end{array}$ & Tholeiite & $\begin{array}{l}\text { Tholeiite } \\
\text { (Oceanite) }\end{array}$ & $\begin{array}{l}\text { Tholeiite } \\
\text { (Oceanite) }\end{array}$ & Tholeiite & Tholeiite & $\begin{array}{c}\text { Tholeiite } \\
\text { (Transitional) }\end{array}$ & $\begin{array}{c}\text { Tholeiite } \\
\text { (Transitional) }\end{array}$ & Tholeiite & Tholeiite & Tholeiite \\
\hline $\mathrm{SiO}_{2}$ & 48.33 & 46.75 & 42.90 & 46.96 & 47.50 & 48.46 & 48.45 & 48.17 & 48.23 & 48.33 & 49.50 \\
\hline $\mathrm{TiO}_{2}$ & 2.95 & 2.74 & 0.95 & 1.78 & 2.45 & 2.39 & 2.31 & 2.24 & 2.25 & 2.34 & 2.24 \\
\hline $\mathrm{Al}_{2} \mathrm{O}_{3}$ & 14.33 & 14.21 & 6.55 & 11.86 & 14.03 & 13.48 & 13.34 & 12.89 & 12.92 & 13.71 & 13.88 \\
\hline $\mathrm{Fe}_{2} \mathrm{O}_{3}(\mathrm{~T})$ & 13.24 & 14.02 & 14.35 & 13.84 & 12.50 & 13.31 & 13.38 & 13.20 & 12.94 & 13.15 & 12.52 \\
\hline $\mathrm{MnO}$ & 0.20 & $\begin{array}{r}14.02 \\
0.17\end{array}$ & 0.16 & $\begin{array}{r}13.04 \\
0.19\end{array}$ & 0.26 & $\begin{array}{r}13.01 \\
0.17\end{array}$ & 0.14 & 0.16 & 0.17 & 0.14 & 0.15 \\
\hline $\mathrm{MgO}$ & 6.88 & 8.70 & 30.50 & 15.93 & 9.47 & 8.65 & 9.55 & 11.20 & 11.31 & 10.15 & 7.30 \\
\hline $\mathrm{CaO}$ & 10.27 & 9.95 & 3.63 & 7.24 & 10.13 & 9.98 & 8.82 & 8.58 & 8.32 & 8.88 & 10.69 \\
\hline $\mathrm{Na}_{2} \mathrm{O}$ & 2.60 & 2.60 & 0.60 & 1.60 & 2.50 & 2.40 & 2.50 & 2.40 & 2.30 & 2.50 & 2.40 \\
\hline $\mathrm{K}_{2} \mathrm{O}$ & 0.65 & 0.41 & 0.04 & 0.10 & 0.44 & 0.33 & 0.51 & 0.35 & 0.32 & 0.22 & 0.42 \\
\hline $\mathrm{P}_{2} \mathrm{O}_{5}$ & 0.34 & 0.35 & 0.10 & 0.19 & 0.31 & 0.32 & 0.26 & 0.22 & 0.24 & 0.27 & 0.26 \\
\hline Total & 99.79 & 99.90 & 99.78 & 99.69 & 99.59 & 99.49 & 99.26 & 99.41 & 99.00 & 99.69 & 99.36 \\
\hline L.O.I. & 2.27 & 1.17 & 7.43 & 6.15 & 4.25 & 2.22 & 10.18 & 5.25 & 4.23 & 5.21 & 3.14 \\
\hline $\mathrm{H}_{2} \mathrm{O}^{+}$ & 0.89 & 1.03 & 6.01 & 4.54 & 1.43 & 1.11 & 1.91 & 2.47 & 2.54 & 2.09 & 0.76 \\
\hline $\mathrm{CO}_{2}$ & 0.19 & 0.21 & 0.41 & 0.31 & 0.45 & 0.12 & 0.26 & 0.26 & 0.30 & 0.24 & 0.21 \\
\hline
\end{tabular}

TABLE 7 - Continued

\begin{tabular}{|c|c|c|c|c|c|c|c|c|c|c|c|c|}
\hline $\begin{array}{l}\text { Sample } \\
\text { (Interval } \\
\text { in } \mathrm{cm} \text { ) }\end{array}$ & $\begin{array}{c}38-1(\# 1 G), \\
76-79\end{array}$ & $\begin{array}{l}38-5 \\
97-100\end{array}$ & $\begin{array}{c}39-5(\# 1 C), \\
102-104\end{array}$ & $\begin{array}{l}40-2(\# 1 \mathrm{G}), \\
111-113\end{array}$ & $\begin{array}{c}\text { 41-1 (\#1C), } \\
19-21\end{array}$ & $\begin{array}{c}42-1(\# 1 G), \\
2-6\end{array}$ & $\begin{array}{c}42-3(\# 1 B), \\
14-16\end{array}$ & $\begin{array}{c}42-5(\# 1 F), \\
37-39\end{array}$ & $\begin{array}{c}45-5(\# 1 A), \\
6-8\end{array}$ & $\begin{array}{l}46-3 \\
72-74\end{array}$ & $\begin{array}{c}47-5(\# 6 B), \\
87-89\end{array}$ & $\begin{array}{l}\text { 49-2 (\#1D), } \\
31-36\end{array}$ \\
\hline Rock Type & Tholeiite & Tholeiite & Tholeiite & Tholeiite & Tholeiite & Tholeiite & Tholeiite & Tholeiite & Tholeiite & Tholeiite & Alkali Basalt & $\begin{array}{c}\text { Tholeiite } \\
\text { (Transitional) }\end{array}$ \\
\hline $\mathrm{SiO}_{2}$ & 46.60 & 47.56 & 48.16 & 47.80 & 48.96 & 49.26 & 48.80 & 48.86 & 48.72 & 48.96 & 45.45 & 46.70 \\
\hline $\mathrm{TiO}_{2}$ & 2.18 & 2.37 & 2.22 & 2.12 & 2.39 & 2.37 & 1.88 & 2.02 & 2.16 & 2.19 & 3.12 & 2.84 \\
\hline $\mathrm{Al}_{2} \mathrm{O}_{3}$ & 13.13 & 14.85 & 13.20 & 12.76 & 14.00 & 13.89 & 16.25 & 15.40 & 14.47 & 14.72 & 14.00 & 15.44 \\
\hline $\mathrm{Fe}_{2} \mathrm{O}_{3}(\mathrm{~T})$ & 13.88 & 13.10 & 13.15 & 13.13 & 13.28 & 12.91 & 11.33 & 11.85 & 12.90 & 13.17 & 14.80 & 14.40 \\
\hline $\mathrm{MnO}$ & 0.17 & 0.15 & 0.16 & 0.16 & 0.12 & 0.13 & 0.13 & 0.14 & 0.21 & 0.13 & 0.19 & 0.16 \\
\hline $\mathrm{MgO}$ & 12.55 & 8.82 & 10.50 & 11.77 & 7.68 & 7.68 & 7.72 & 6.98 & 6.69 & 6.75 & 6.76 & 6.19 \\
\hline $\mathrm{CaO}$ & 8.50 & 9.97 & 9.71 & 9.29 & 10.07 & 10.14 & 10.26 & 10.94 & 11.15 & 9.58 & 10.92 & 9.77 \\
\hline $\mathrm{Na}_{2} \mathrm{O}$ & 2.30 & 2.40 & 2.10 & 2.10 & 2.60 & $\begin{array}{r}10.14 \\
2.70\end{array}$ & 2.70 & 2.50 & 2.60 & 2.80 & 2.60 & 2.90 \\
\hline $\mathrm{K}_{2} \mathrm{O}$ & 0.45 & 0.18 & 0.22 & 0.20 & 0.24 & 0.25 & 0.25 & 0.23 & 0.18 & 0.65 & 0.89 & 0.52 \\
\hline $\mathrm{P}_{2} \mathrm{O}_{5}$ & 0.23 & 0.27 & 0.25 & 0.24 & 0.26 & 0.26 & 0.24 & 0.23 & 0.21 & 0.26 & 0.33 & 0.32 \\
\hline Total & 99.99 & 99.67 & 99.67 & 99.57 & 99.60 & 99.59 & 99.56 & 99.15 & 99.29 & 99.21 & 99.06 & 99.24 \\
\hline L.O.I. & 3.44 & 4.05 & 2.16 & 2.36 & 3.08 & 2.89 & 2.62 & 2.33 & 2.26 & 3.60 & 4.65 & 3.97 \\
\hline $\mathrm{H}_{2} \mathrm{O}^{+}$ & $\begin{array}{l}3.44 \\
2.49\end{array}$ & $\begin{array}{l}4.05 \\
2.21\end{array}$ & $\begin{array}{l}2.10 \\
1.58\end{array}$ & $\begin{array}{l}2.50 \\
2.09\end{array}$ & $\begin{array}{l}3.08 \\
1.08\end{array}$ & $\begin{array}{l}2.89 \\
1.02\end{array}$ & $\begin{array}{l}2.62 \\
1.31\end{array}$ & $\begin{array}{l}2.33 \\
0.88\end{array}$ & $\begin{array}{l}2.20 \\
0.81\end{array}$ & $\begin{array}{l}3.00 \\
1.24\end{array}$ & $\begin{array}{l}4.03 \\
1.74\end{array}$ & 1.84 \\
\hline $\mathrm{CO}_{2}$ & 0.27 & 0.24 & 0.18 & 0.13 & 0.20 & 0.22 & 0.21 & 0.30 & 0.32 & 0.19 & 1.06 & 0.22 \\
\hline
\end{tabular}


TABLE 8

Hole 433C, Suiko Seamount: Trace Elements

\begin{tabular}{|c|c|c|c|c|c|c|c|c|c|c|c|c|c|c|c|c|c|c|c|c|c|c|c|c|c|c|c|}
\hline \multirow{2}{*}{$\begin{array}{c}\text { Sample } \\
\text { (Interval in } \mathrm{cm} \text { ) }\end{array}$} & \multirow{2}{*}{$\begin{array}{c}\mathrm{Sc} \\
\text { NAA }\end{array}$} & \multirow{2}{*}{$\begin{array}{c}\mathrm{Ti} \\
\mathrm{XRF}\end{array}$} & \multirow{2}{*}{$\begin{array}{c}\mathrm{V} \\
\mathrm{XRF}\end{array}$} & \multirow{2}{*}{$\underset{\mathrm{XRF}}{\mathrm{Cr}}$} & \multirow{2}{*}{$\begin{array}{l}\mathrm{Mn} \\
\mathrm{XRF}\end{array}$} & \multirow{2}{*}{$\begin{array}{c}\mathrm{Fe} \\
\mathrm{XRF}\end{array}$} & \multicolumn{2}{|c|}{$\mathrm{Co}$} & \multicolumn{2}{|c|}{$\mathrm{Ni}$} & & $\mathrm{zb}$ & & & & $\mathrm{Nb}$ & & & B & a & & Eu & & & & & \\
\hline & & & & & & & XRF & NAA & XRF & NAA & $\mathrm{XRF}$ & NAA & XRF & $\mathrm{XRF}$ & $\mathrm{XRF}$ & $\mathrm{XRF}$ & NAA & NAA & XRF & NAA & NAA & NAA & NAA & NAA & NAA & NAA & NAA \\
\hline $4-1,39-41$ & 30.5 & 17520 & 338 & 197 & 1470 & 104510 & & 51.3 & 122 & 127 & 13.4 & 11.5 & 371 & 34.9 & 203 & 26.3 & 0.04 & 0.07 & 161 & 204 & 18.4 & 2.19 & 0.92 & 4.81 & 1.79 & 1.71 & 0.47 \\
\hline & 25.7 & & 261 & 752 & 1160 & 99120 & 69 & 72.1 & 516 & 517 & 5.2 & & 162 & 23.6 & 114 & 10.9 & 0.02 & 0.04 & & 177 & & 1.35 & & & 0.75 & 0.8 & 0.25 \\
\hline & 27.3 & 11460 & 265 & 664 & 1010 & 102410 & 65 & 63.1 & 401 & 410 & 3.5 & 5 & 21 & 23.7 & 118 & 12.1 & 0.09 & 0.02 & & 79 & 8.9 & 14.1 & 0.60 & 6 & 0.78 & 0.77 & 0.08 \\
\hline 07 & 29 & 11820 & 277 & 575 & 1160 & 104650 & 58 & 57.6 & 329 & 304 & 6.0 & 6.4 & 235 & 26.5 & 120 & 12.1 & 0.13 & 0.04 & & 171 & 9.1 & 1.46 & 0.63 & & 0.84 & 0.85 & 0.24 \\
\hline $10-4,118-121$ & 18 & 6900 & 169 & 1328 & 1470 & 98700 & 100 & 104 & 1391 & 1208 & 5.1 & 4.3 & 102 & 14.0 & 70 & 6.6 & 0.02 & 0.07 & & 34 & 4.8 & 0.8 & 0.34 & 1.60 & 0.43 & 0.48 & 0.19 \\
\hline $\begin{array}{l}10-5,118-120 \\
10-1,7-9\end{array}$ & $\begin{array}{l}27.7 \\
25.7\end{array}$ & $\begin{array}{l}11340 \\
10200\end{array}$ & $\begin{array}{l}242 \\
244\end{array}$ & $\begin{array}{l}427 \\
752\end{array}$ & $\begin{array}{r}540 \\
1240\end{array}$ & $\begin{array}{r}109900 \\
99050\end{array}$ & $\begin{array}{l}64 \\
73\end{array}$ & $\begin{array}{l}61.2 \\
71.5\end{array}$ & $\begin{array}{l}270 \\
520\end{array}$ & & $\begin{array}{l}9.8 \\
5.8\end{array}$ & $\begin{array}{r}11.4 \\
7.2\end{array}$ & $\begin{array}{l}165 \\
179\end{array}$ & $\begin{array}{l}21.5 \\
22.4\end{array}$ & 117 & 10.3 & 0.02 & $\begin{array}{l}0.09 \\
0.02\end{array}$ & & 59 & $\begin{array}{l}7.8 \\
8.4\end{array}$ & 1.47 & $\begin{array}{l}0.60 \\
0.57\end{array}$ & & $\begin{array}{l}0.70 \\
0.72\end{array}$ & 0.68 & 0.42 \\
\hline & $\begin{array}{l}20.1 \\
29.6\end{array}$ & 11340 & $\begin{array}{l}4 \\
8\end{array}$ & 375 & $\begin{array}{l}1240 \\
1390\end{array}$ & 93450 & $\begin{array}{l}73 \\
52\end{array}$ & 52. & $\begin{array}{l}52 \\
16\end{array}$ & & $\begin{array}{l}5.8 \\
2.5\end{array}$ & & $\begin{array}{l}17 \\
26\end{array}$ & $\begin{array}{l}22.4 \\
25.5\end{array}$ & $\begin{array}{l}103 \\
123\end{array}$ & $\begin{array}{l}11.4 \\
10.5\end{array}$ & & $\begin{array}{l}0 . \\
0 .\end{array}$ & & $\begin{array}{l}60 \\
86\end{array}$ & & & & & & 11 & $\begin{array}{l}0.13 \\
0.25\end{array}$ \\
\hline & 30.1 & 11520 & 269 & 407 & & 91210 & 56 & 54. & 188 & 187 & 2.7 & & 29 & 25.8 & 124 & 9.7 & & & & 38 & 8.8 & 1.47 & 0.63 & & 0.73 & 0.75 & 0.16 \\
\hline $11-4,110-112$ & 34.3 & 14400 & 326 & 329 & 850 & 88970 & 46 & 46.6 & 124 & 129 & 11.2 & 11.4 & 305 & 30.9 & 145 & 13.8 & 0.01 & 0.21 & & 51 & 10.6 & 1.90 & 0.82 & 3.68 & 0.97 & 0.90 & 0.22 \\
\hline $12-3$ & 32.3 & 14100 & 314 & 279 & 1080 & 86100 & 47 & 48.3 & 107 & 112 & 0.4 & & 304 & .7 & 147 & 14.4 & & & & 71 & 9.8 & 1.77 & 0.80 & 2.73 & 0.97 & 0.86 & 0.24 \\
\hline 5 & $\begin{array}{l}34.2 \\
34.0\end{array}$ & $\begin{array}{l}13560 \\
16080\end{array}$ & $\begin{array}{l}328 \\
322\end{array}$ & $\begin{array}{r}232 \\
73\end{array}$ & $\begin{array}{r}1320 \\
700\end{array}$ & $\begin{array}{l}92120 \\
97790\end{array}$ & $\begin{array}{l}48 \\
49\end{array}$ & $\begin{array}{l}46.7 \\
45.7\end{array}$ & $\begin{array}{r}100 \\
53\end{array}$ & $\begin{array}{l}9 \\
4\end{array}$ & 4 & $\begin{array}{l}4.4 \\
8.5\end{array}$ & & & 143 & & 0.02 & & & $\begin{array}{r}103 \\
86\end{array}$ & 10.9 & & & & & & 0.22 \\
\hline & 32.4 & 15180 & 320 & 77 & 1080 & 9485 & 47 & 46. & 48 & 47 & 8 & & 30 & & $\begin{array}{l}163 \\
158\end{array}$ & $\begin{array}{l}15.3 \\
14.3\end{array}$ & & & & $\begin{array}{l}0 \\
5\end{array}$ & 9.9 & 83 & 0.82 & & & & $\begin{array}{l}0.31 \\
0.15\end{array}$ \\
\hline $15-1,115-118$ & 33.4 & 15900 & 328 & 65.0 & 930 & 100240 & 51 & 48.7 & 45 & 48 & 2 & & 296 & 35.4 & 160 & 14.5 & & & & 65 & 10.4 & 2.11 & 0.90 & 4.06 & 1.01 & 0.92 & 0.39 \\
\hline & 31.8 & 5240 & 310 & 69.5 & 850 & 8160 & 49 & 45.9 & 50 & 55 & 18 & 17.6 & 242 & 27.8 & 159 & 13.3 & 0.02 & 0.19 & & 54 & 10.4 & 1.81 & 0.78 & 3.77 & 0.92 & 0.84 & 0.26 \\
\hline & & & & 75.3 & 1390 & & 52 & & 54 & 59 & 1.5 & & 30 & & & & & & & & & & & & & & \\
\hline & & 14700 & 301 & 128 & 10 & & 46 & 45 & 80 & 76 & 1.9 & & 301 & 32.8 & 154 & 12.3 & & & & 45 & 9. & & 0.8 & & 0 & 0.79 & 0.22 \\
\hline & & & & & & & & & & & 1.6 & & & & & & & & & & & & & & & & \\
\hline & 31.4 & 16980 & 308 & 190 & 850 & 86241 & 47 & 46.6 & 98 & 94 & 0.4 & 2.6 & 35 & 35.6 & 179 & 15.9 & 0.02 & & & 91 & 11.5 & 2.20 & 0.97 & 4.38 & 1.09 & 1.02 & 0.14 \\
\hline & 28 & 16260 & 276 & 176 & 850 & 90440 & 49 & 48.3 & 126 & 130 & 2.1 & & 353 & 32.7 & 170 & 14.4 & 0.02 & 0.01 & & 87 & 11.6 & 1.91 & 0.87 & 4.42 & 1.07 & 0.95 & 0.27 \\
\hline & & & & 16 & & & & & 11 & 15 & 2.6 & 4.4 & & & & & & & & & 13. & & & & & & \\
\hline & & & & 26 & 1390 & 11 & & & 276 & 276 & 18.2 & 18.2 & & & 138 & 11.4 & 0.03 & & & & & & & & 32 & 0.72 & 0.21 \\
\hline & & & & 70 & 62 & & 63 & 64 & 489 & 468 & 2.6 & 3.8 & & & 95 & 7.4 & & & & & & & & & 0.048 & 0.45 & 0.12 \\
\hline $24-1,12$ & 21 & 7980 & 183 & 875 & 1160 & 99680 & 92 & 91.2 & 1049 & 1014 & 2.1 & & 114 & 18.2 & 79 & 5.5 & & 0.06 & & & 4.6 & .83 & 0.43 & 1.77 & 0.35 & 26 & \\
\hline $\begin{array}{l}24-7,141-144 \\
25-2,22-25\end{array}$ & 19.6 & & & & $\begin{array}{l}1320 \\
1080\end{array}$ & & $\begin{array}{l}96 \\
54\end{array}$ & $\begin{array}{l}97 \\
53\end{array}$ & 1068 & 1139 & & 15 & & & & 5.3 & & 0.07 & & 44 & 3.4 & 0.92 & 0.41 & 1.63 & 0.35 & 32 & \\
\hline & & & & & & & & & $\begin{array}{l}15 \\
11\end{array}$ & $\begin{array}{l}15 \\
10\end{array}$ & $\begin{array}{l}1.2 \\
1.2\end{array}$ & & & & & & & & & & & & & & & $\begin{array}{l}65 \\
61\end{array}$ & \\
\hline & 27 & 2600 & & 596 & & & & & 438 & 41 & 1.6 & 2.2 & & & 12 & .0 & 0.02 & 0.04 & & & $\begin{array}{l}8.2 \\
7.5\end{array}$ & & & & 12 & 0.57 & $\begin{array}{l}0.11 \\
0.10\end{array}$ \\
\hline-11 & 30. & 12 & & & 116 & 93240 & 59 & 55.8 & 232 & 230 & & 2. & 26 & & 145 & & 0.02 & & & & 8.9 & .80 & 0.78 & & .82 & 0.71 & 0.17 \\
\hline 134 & 11.1 & & 145 & 1186 & 1390 & 102690 & 109 & 111 & 1335 & 1434 & 3.7 & 2.6 & 89 & 14.8 & 63 & 6 & 0.01 & 0.11 & & 9 & & 0.84 & 37 & 1.16 & 0.31 & 0.35 & \\
\hline & & & & 14 & & & 47 & 47 & & & & & & & & & & & & & & & & & & & \\
\hline & & & & 13 & & & 45 & 45 & $\begin{array}{l}68 \\
70\end{array}$ & 6 & & & & & & & & & & & & & & & & & 26 \\
\hline & $\begin{array}{l}28.2 \\
28.2\end{array}$ & $\begin{array}{l}17700 \\
16440\end{array}$ & $\begin{array}{l}334 \\
264\end{array}$ & $\begin{array}{l}167 \\
206\end{array}$ & $\begin{array}{l}1550 \\
1320\end{array}$ & $\begin{array}{l}92680 \\
98140\end{array}$ & $\begin{array}{l}45 \\
58\end{array}$ & & $\begin{array}{r}19 \\
184\end{array}$ & $\begin{array}{r}80 \\
201\end{array}$ & & $\begin{array}{l}8.8 \\
3.4\end{array}$ & & & & & & & & & & 55 & & & & $\begin{array}{l}1.02 \\
0.84\end{array}$ & $\begin{array}{l}0.12 \\
0.21\end{array}$ \\
\hline 10-198 & 14.6 & 5700 & 129 & 1100 & 1240 & 100450 & 117 & 112 & 1614 & 1628 & 1.5 & & 5 & & 59 & .4 & & & & & 3.4 & 57 & .27 & & 77 & 0.24 & .07 \\
\hline & & & & & & & & & & & & 2.5 & & & & & & & & & & & & & & & \\
\hline & & & & & & & 55 & & & & & & & & & & & & & & & & & & & & \\
\hline & & & & & & & & & & & & & & & & & & & & & 18 & & & & & & \\
\hline $36-2,16-19$ & 29.7 & 13860 & 274 & 522 & 1080 & 93660 & 50 & 52.4 & 287 & 285 & 4. & 3.9 & 251 & 32.2 & 145 & 11. & 0.03 & 0.0 & & 66 & 9.5 & 1.73 & .82 & & 0.87 & 0.77 & 0.25 \\
\hline & & & 273 & 592 & & & 57 & & & & 3. & 4.1 & & & 147 & 12.7 & 0.02 & & & 48 & 7.8 & 1.75 & .77 & 3.63 & 0.85 & 0.75 & 0.22 \\
\hline & & & & & & & & & & & & & & & & & & 0.03 & & & & & & & & & \\
\hline & & & & & & & & & & & & & & & & & & & & & & & & & & & \\
\hline & & & & & & & & & & & & & & & & & & & & & & & & & & & 0.24 \\
\hline $38-1,76-79$ & 26.7 & 13080 & 254 & 452 & 1320 & 97160 & 67 & 68.2 & 448 & 491 & 4.2 & 2.9 & 268 & 26.0 & 137 & 11.3 & 0.02 & 0.06 & & 50 & 5.6 & 1.63 & .63 & 3.35 & 0.75 & 0.66 & \\
\hline & $\begin{array}{l}28.8 \\
28.4\end{array}$ & & & & 1160 & & $\begin{array}{l}52 \\
56\end{array}$ & $\begin{array}{l}51.4 \\
56\end{array}$ & & 156 & 0.2 & & $\begin{array}{l}293 \\
263\end{array}$ & & & 13.9 & 0.03 & 00 & & 54 & $\begin{array}{l}9 \\
85\end{array}$ & 1.73 & .76 & $\begin{array}{l}3.68 \\
3.52\end{array}$ & $\begin{array}{l}0.85 \\
0.80\end{array}$ & 0.68 & $\begin{array}{l}0.14 \\
0.18\end{array}$ \\
\hline & & & & & & & & & & & & & & & & & & & & & & & & & & & \\
\hline & 33.7 & & & & & & & 46. & 87 & 85 & & 2.2 & & & & & & & & & & & & & & 0.72 & 0.18 \\
\hline & & & & 215 & 1010 & & & & 86 & & 1.9 & & 277 & 30.4 & 136 & 11.3 & 0.02 & & & 20 & 8.1 & 1.71 & .77 & & 0.77 & 0.63 & 0.18 \\
\hline & & & & & & & 43 & & & & & & & & 108 & 8.2 & & & & & 5.5 & & .64 & & 8 & 0.51 & 0.16 \\
\hline & & & & & & & & & & & & & & & & & & & & & & & & & & & \\
\hline & & & & & & & & & & & & & & & & & & & & & & & & & & & \\
\hline & & & & & & & & & & & & & & & & & & & & & & & & & & & \\
\hline & & & & & & & & & & 19 & 12. & & & & 19 & 14.7 & & 0.43 & & & & & & & & & \\
\hline $49-2,31-36$ & 25.4 & 17040 & 290 & 205 & 1240 & 100800 & 51 & 41.2 & 138 & 93 & 2.9 & & 338 & 34.1 & 175 & & 0.02 & & & 98 & 10.9 & 1.94 & 0.89 & 3.88 & 0.98 & 0.94 & 0.17 \\
\hline
\end{tabular}


P. CAMBON, J. L. JORON, H. BOUGAULT, M. TREUIL

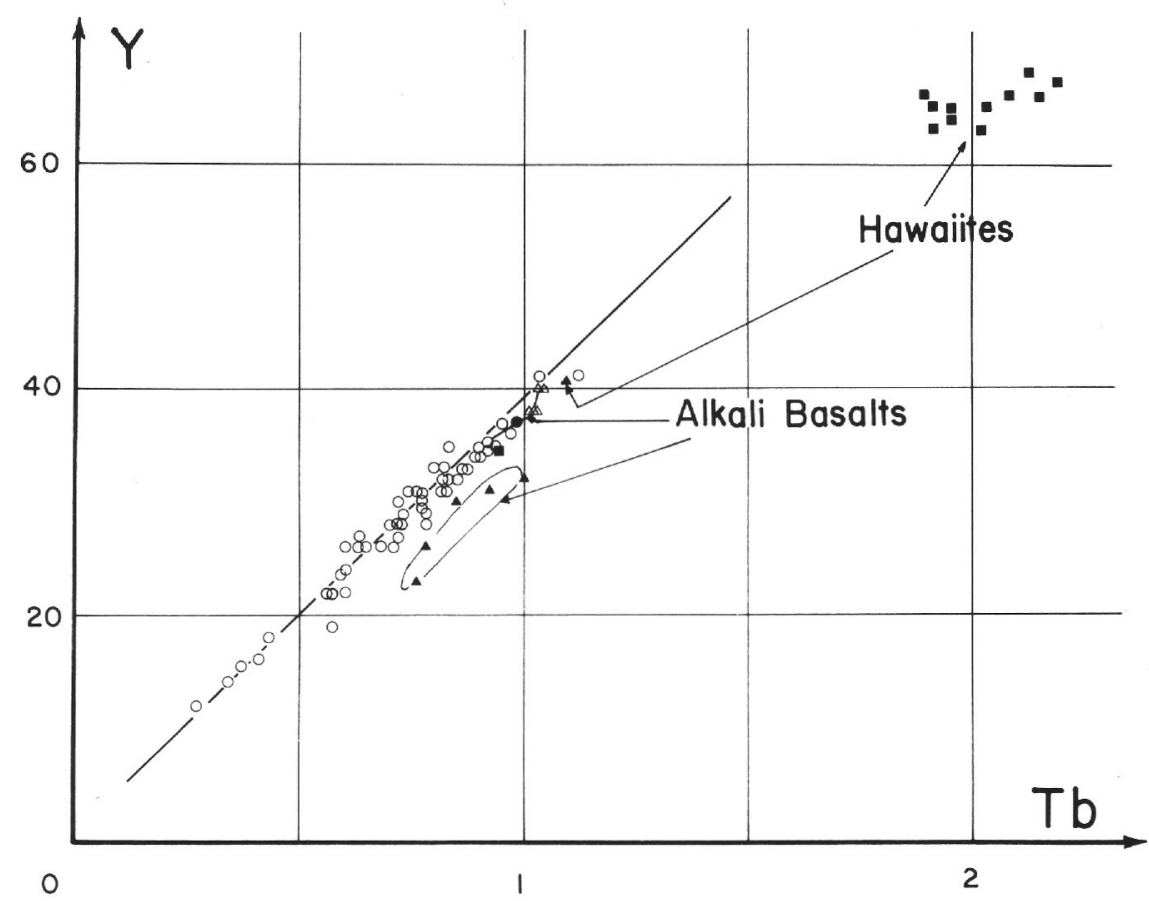

Figure 1. Y versus Tb: black squares correspond to samples from Hole 430A, black triangles to Hole $432 \mathrm{~A}$, open triangles to Hole $433 \mathrm{~A}$, black dot to Hole 433B, and open circle to Hole 433C. Alkali basalts belonging respectively to Sites 432 and 433 are delimited. All open circles (433C) except one sample (alkali basalt) are tholeiites or transitional tholeiites, including oceanites and ankaramite. One sample from Hole $330 \mathrm{~A}$ (black squares) is a tholeiite. 


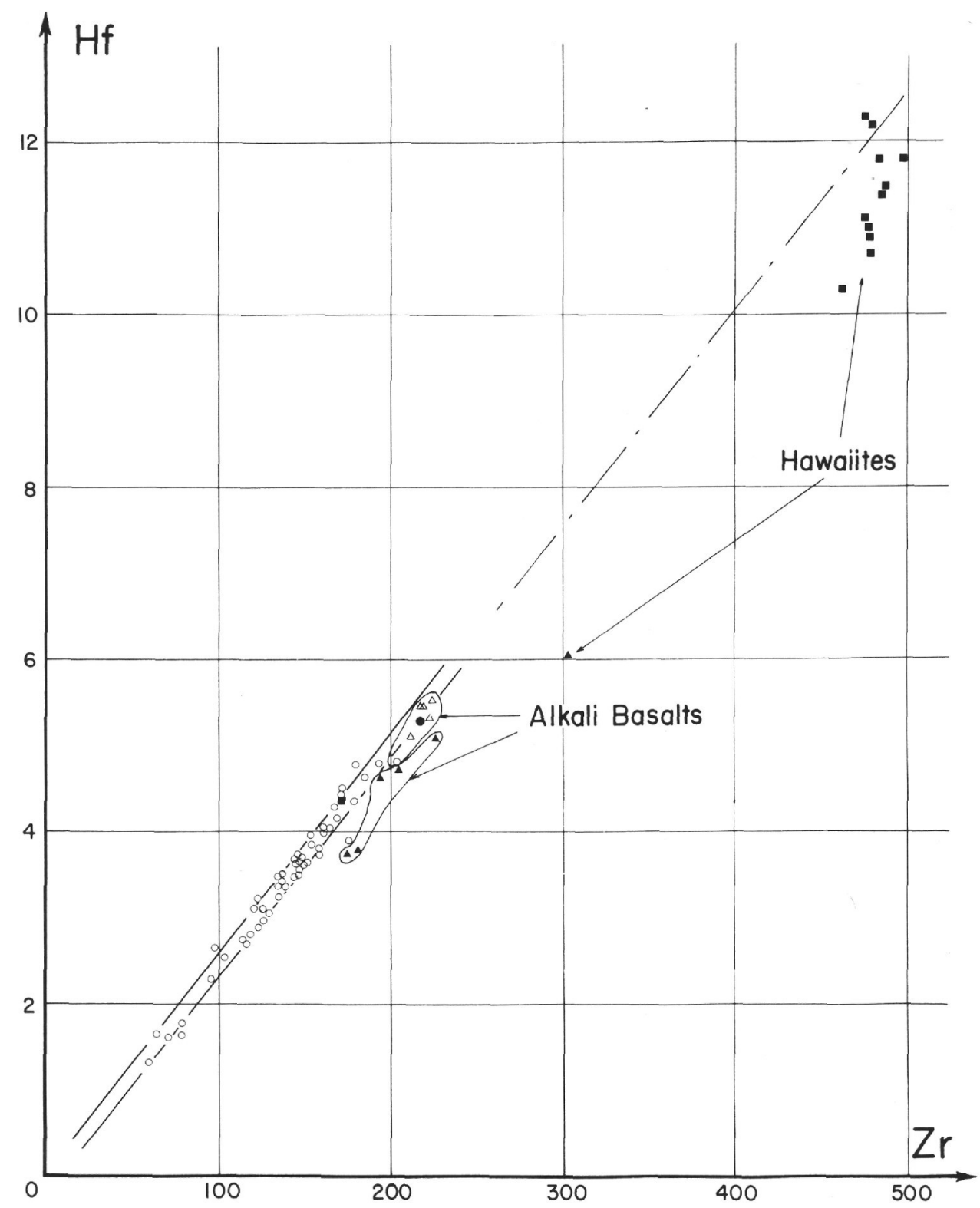

Figure 2. Hf versus Zr: same symbols as in Figure 1. 


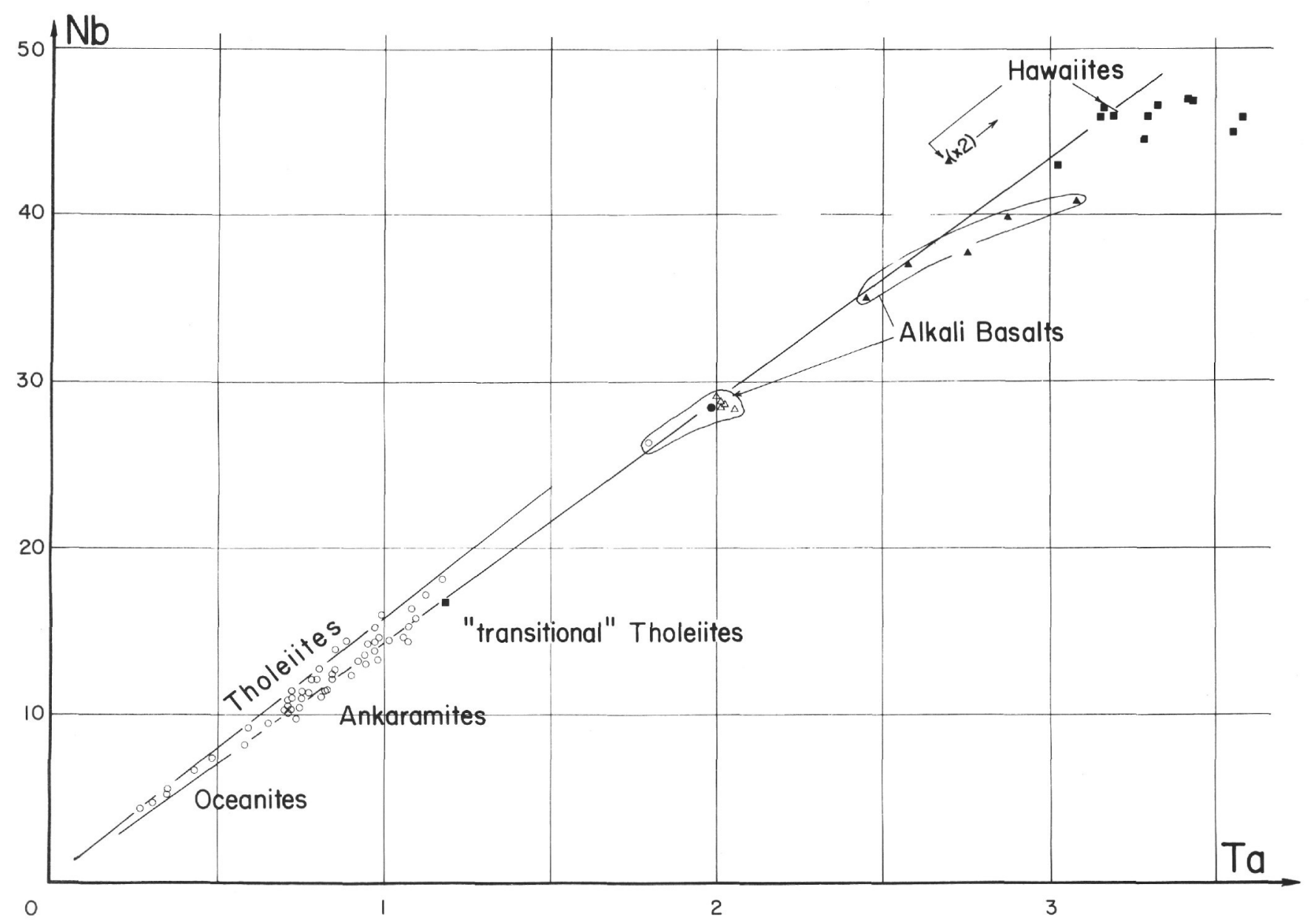

Figure 3. Nb versus Ta: same symbols as in Figure 1. The sample mentioned $(\times 2)$ has concentrations twice higher than corresponding to the plot. 


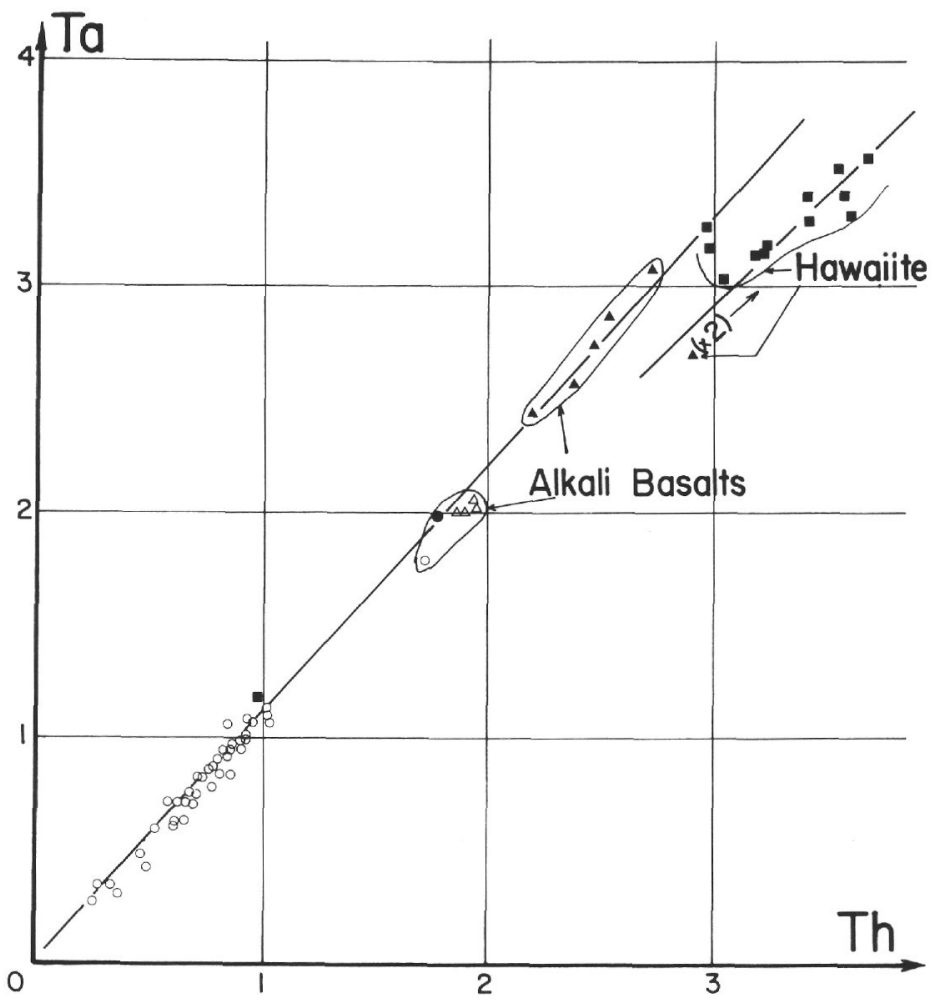

Figure 4. Ta versus Th: same symbols as in Figure 1. The sample mentioned $(\times 2)$ has concentrations twice higher than corresponding to the plot.

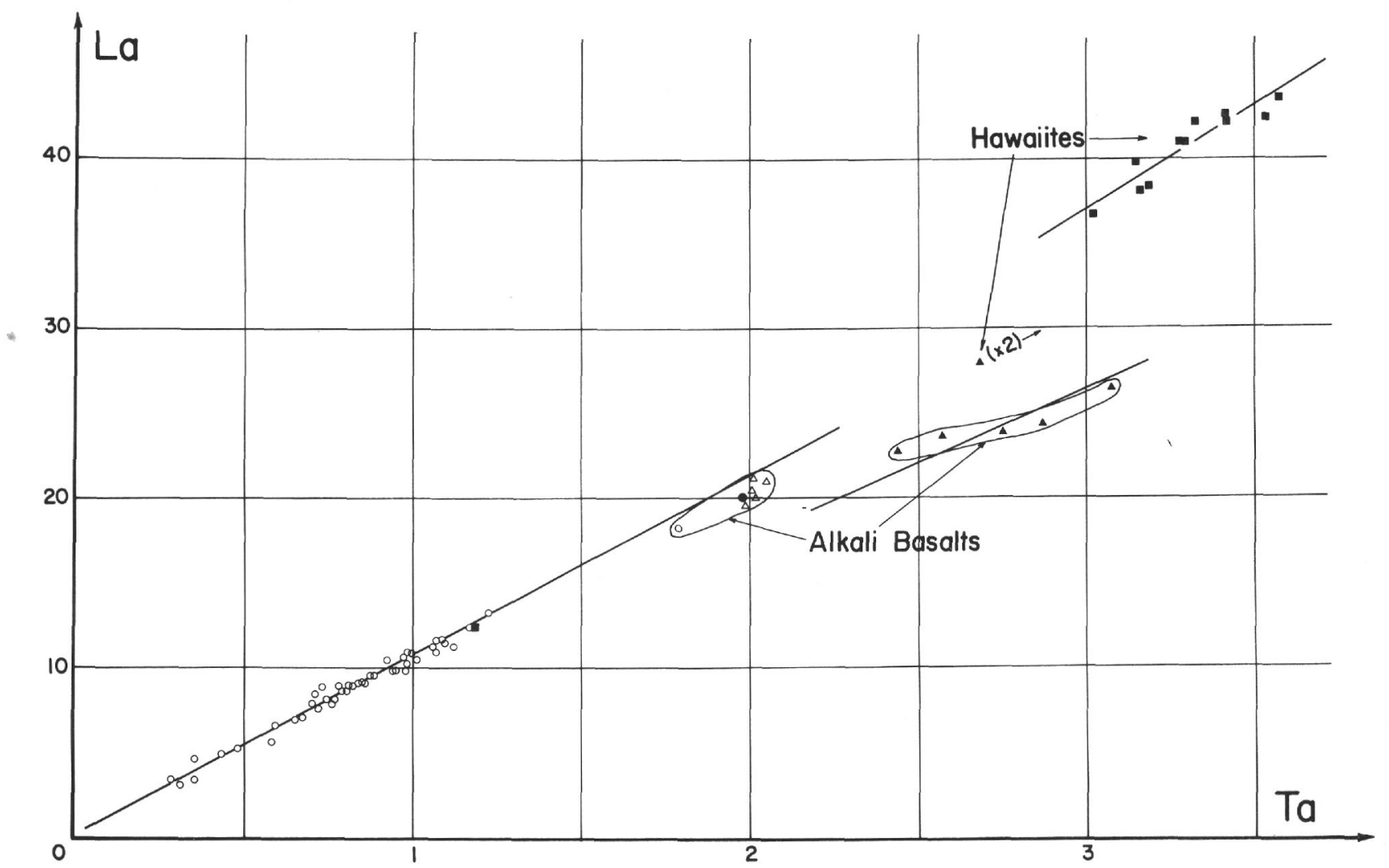

Figure 5. La versus Ta: same symbols as in Figure 1. The sample mentioned $(\times 2)$ has concentrations twice higher than corresponding to the plot. 
P. CAMBON, J. L. JORON, H. BOUGAULT, M. TREUIL

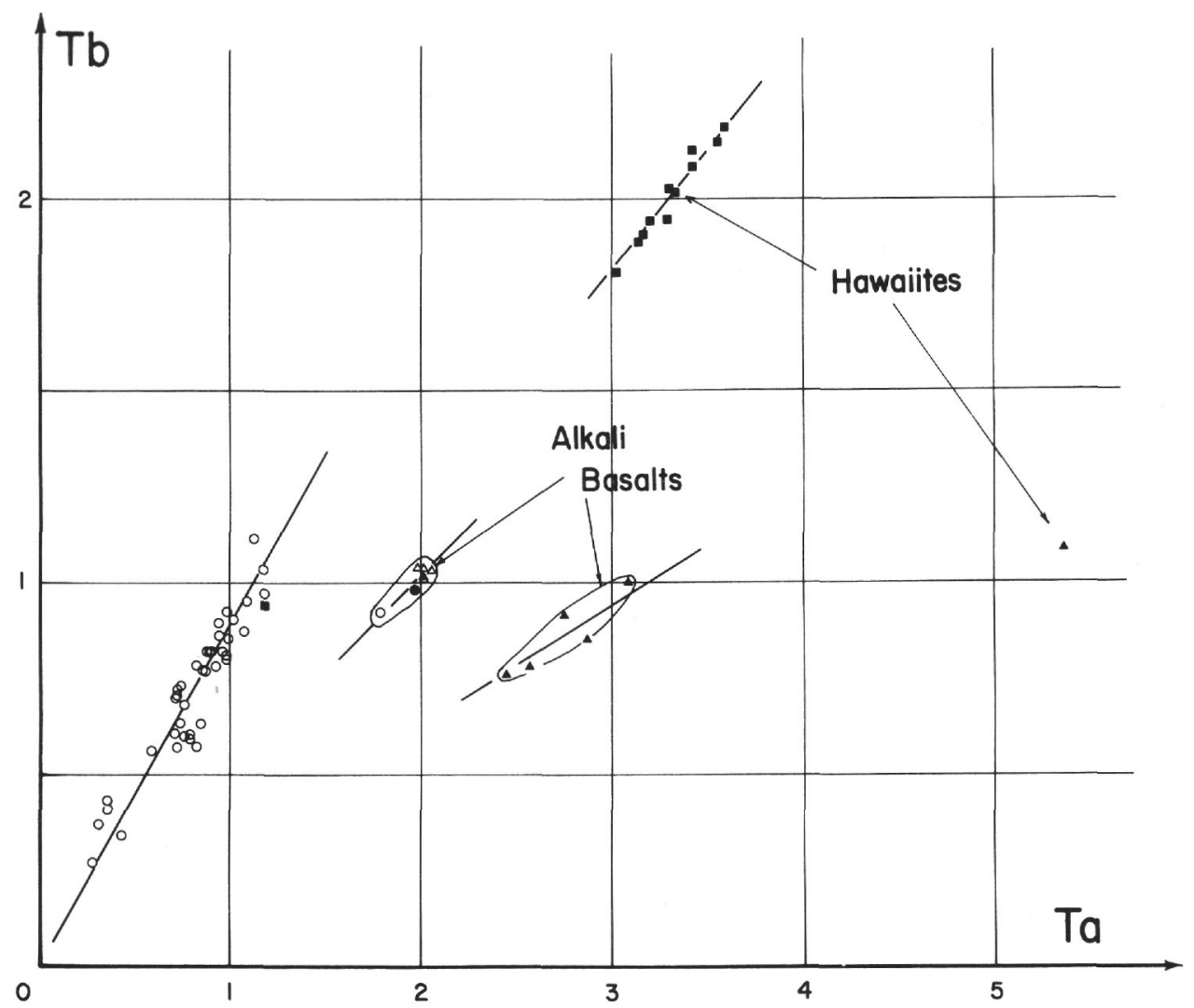

Figure 6. Tb versus Ta: same symbols as in Figure 1.

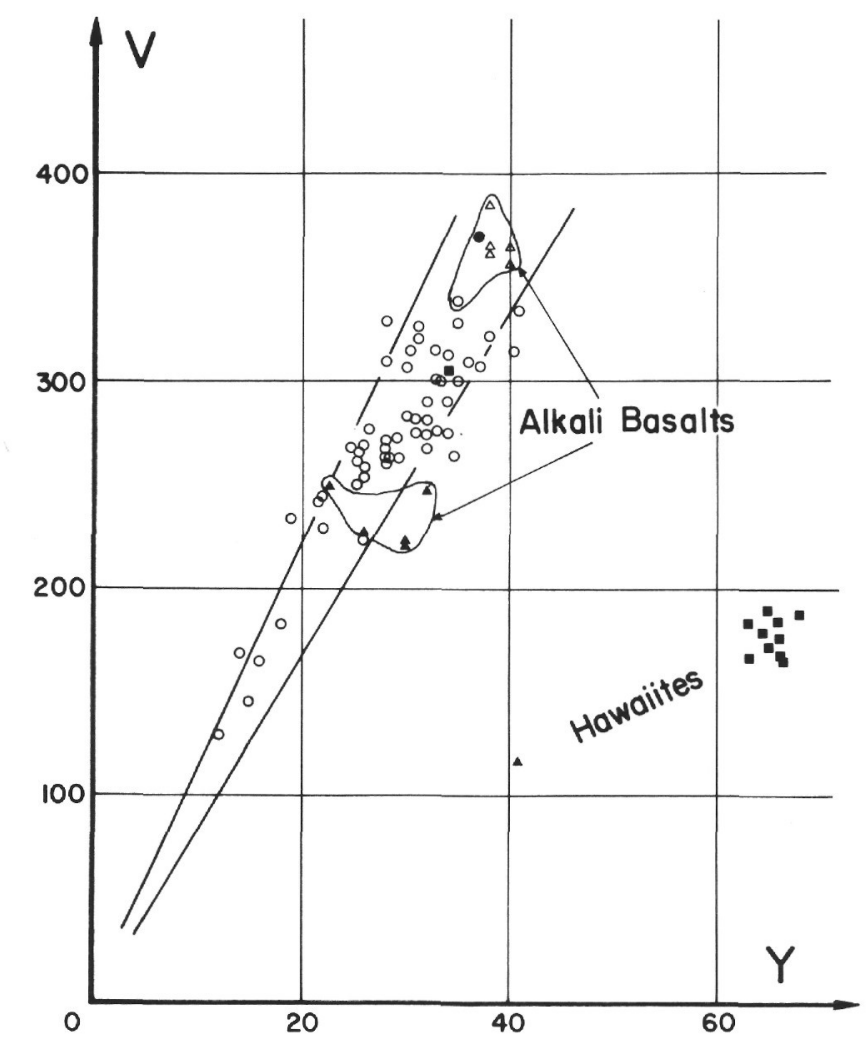

Figure 7. V versus Y: same symbols as in Figure 1. 


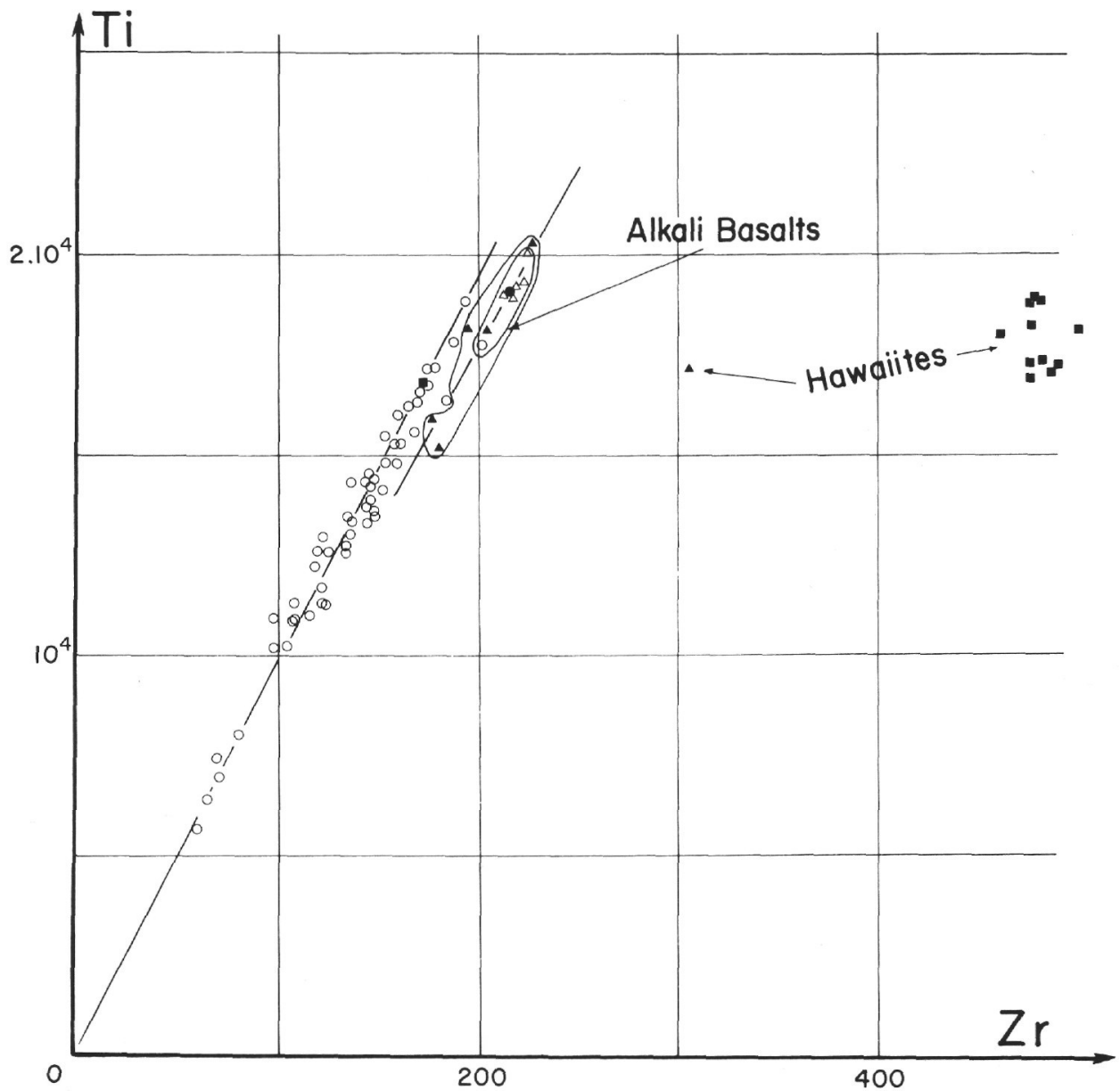

Figure 8. Ti versus Zr: same symbols as in Figure 1. 